Yukawa Institute Kyoto

YITP-00-24

hep-th/0005277

May 2000

\title{
Quantum Calogero-Moser Models: Integrability for all Root Systems
}

\author{
S. P. Khastgir, A. J. Pocklington and R. Sasaki \\ Yukawa Institute for Theoretical Physics, Kyoto University, \\ Kyoto 606-8502, Japan
}

\begin{abstract}
The issues related to the integrability of quantum Calogero-Moser models based on any root systems are addressed. For the models with degenerate potentials, i.e. the rational with/without the harmonic confining force, the hyperbolic and the trigonometric, we demonstrate the following for all the root systems: (i) Construction of a complete set of quantum conserved quantities in terms of a total sum of the Lax matrix $L$, i.e. $\sum_{\mu, \nu \in \mathcal{R}}\left(L^{n}\right)_{\mu \nu}$, in which $\mathcal{R}$ is a representation space of the Coxeter group. (ii) Proof of Liouville integrability. (iii) Triangularity of the quantum Hamiltonian and the entire discrete spectrum. Generalised Jack polynomials are defined for all root systems as unique eigenfunctions of the Hamiltonian. (iv) Equivalence of the Lax operator and the Dunkl operator. (v) Algebraic construction of all excited states in terms of creation operators. These are mainly generalisations of the results known for the models based on the $A$ series, i.e. su( $N)$ type, root systems.
\end{abstract}




\section{Introduction}

Calogero-Moser models are one-dimensional dynamical systems with long range interactions having a remarkable property that they are integrable at both classical and quantum levels. In fact the integrability or more precisely the triangularity of the quantum Hamiltonian was first discovered by Calogero [1] for the model with inverse square potential plus a confining harmonic force and by Sutherland [2] for the particles on a circle with the inverse square potential. Later classical integrability of the models in terms of Lax pairs was proved by Moser [3]. Olshanetsky and Perelomov [4] showed that these models were based on $A_{r}$ root systems and generalisations of the models based on other root systems including the non-crystallographic ones were introduced [5].

In this paper we discuss quantum Calogero-Moser models with degenerate potentials, that is the rational with/without harmonic force, the hyperbolic and the trigonometric potentials based on all root systems. We demonstrate, based on previous works of universal Lax pairs for classical [6, 7, 8] and quantum models [9], that various results known for the quantum $A_{r}$ models can be generalised to the models based on any root systems as well. They are (i) Construction of a complete set of quantum conserved quantities in terms of quantum Lax pairs and other methods. (ii) Universal proof of Liouville integrability for the rational, hyperbolic and trigonometric potential models. Namely, the quantum conserved quantities commute among themselves. (iii) Triangularity of the quantum Hamiltonian is demonstrated explicitly for all the models. In other words, the Hamiltonian is shown, in certain bases, to be decomposed into a sum of finite dimensional triangular matrices. Thus any eigenvalue equation can be solved by finite steps of linear algebraic processes only. This also gives the entire discrete spectrum of the models. As unique eigenfunctions of the Hamiltonian, generalisation of Jack polynomials and multivariable Laguerre (Hermite) polynomials are defined for all root systems. (iv) Equivalence of the quantum Lax pair method and that of so-called differential-reflection (Dunkl) operators [10] is demonstrated. (v) For rational models with harmonic confining force, an algebraic construction of all excited states in terms of creation (annihilation) operators is achieved.

For the $A_{r}$ models, the Lax pairs, conserved quantities and their involution were discussed by many authors with varied degrees of completeness and rigour, see for example, [5], [11] [20]. The point (iv) was shown by Wadati and collaborators [18 and point (v) was initiated by Perelomov [12] and developed by Brink and collaborators [17] and Wadati 
and collaborators [18]. A rather different approach by Heckman and Opdam [21, 22] to Calogero-Moser models with degenerate potentials based on any root systems should also be mentioned in this connection.

For the general background and the motivations of this series of papers and the physical applications of the Calogero-Moser models with various potentials to lower dimensional physics, ranging from solid state to particle physics and supersymmetric Yang-Mills theory, we refer to our previous papers [6, [7] and references therein.

This paper is organised as follows. In section two, quantum Calogero-Moser Hamiltonian with degenerate potentials is introduced as a factorised form (2.5). Connection with root systems and the Coxeter invariance is emphasised. Some rudimentary facts of the root systems and reflections are summarised in Appendix A. A universal Coxeter invariant ground state wavefunction together with its energy eigenvalue are presented. In section three we show that all the excited states are also Coxeter invariant and that the Hamiltonian is triangular in certain bases. Complete sets of quantum conserved quantities are derived from quantum Lax operator $L$ in section four. Instead of the trace, the total sum of $L^{n}$ is conserved. That is $\operatorname{Ts}\left(L^{n}\right)=\sum_{\mu, \nu \in \mathcal{R}}\left(L^{n}\right)_{\mu \nu}$, in which $\mathcal{R}$ is a representation space of the Coxeter group. The details of the complete set for each root system are given in Appendix B. In section five the creation and annihilation operators for the rational models with harmonic force are derived. In section six, the equivalence of the Lax pair operator formalism and the socalled differential-reflection (Dunkl) operators is demonstrated and the quantum conserved quantities are expressed in terms of the latter. In section seven an algebraic construction of excited states in terms of the differential-reflection (Dunkl) operators for rational models with harmonic force is presented. The complete sets of explicit eigenfunctions for the rank two models are derived in terms of separation of variables based on the Coxeter invariant variables. Section eight gives a universal proof of the Liouville integrability for models with rational (without the confining force), hyperbolic and trigonometric potentials. For rational models with harmonic force, the involution is demonstrated for those based on classical root systems. A simple use of the quantum Lax pair with spectral parameter is mentioned. The final section is for summary and comments. 


\section{Quantum Calogero-Moser Models}

In this section we briefly introduce the quantum Calogero-Moser models along with appropriate notation and background for the main body of this paper. A Calogero-Moser model is a Hamiltonian system associated with a root system $\Delta$ of rank $r$, which is a set of vectors in $\mathbf{R}^{r}$ with its standard inner product. A brief review of the properties of the root systems and the associated reflections will be found in the Appendix A.

\subsection{Factorised Hamiltonian}

The dynamical variables of the Calogero-Moser model are the coordinates $\left\{q_{j}\right\}$ and their canonically conjugate momenta $\left\{p_{j}\right\}$, with the canonical commutation relations:

$$
\left[q_{j}, p_{k}\right]=i \delta_{j k}, \quad\left[q_{j}, q_{k}\right]=\left[p_{j}, p_{k}\right]=0, \quad j, k=1, \ldots, r .
$$

These will be denoted by vectors in $\mathbf{R}^{r}$

$$
q=\left(q_{1}, \ldots, q_{r}\right), \quad p=\left(p_{1}, \ldots, p_{r}\right) .
$$

The momentum operator $p_{j}$ acts as

$$
p_{j}=-i \frac{\partial}{\partial q_{j}}, \quad j=1, \ldots, r .
$$

As for the interactions we consider only the degenerate potentials, that is the rational (with/without harmonic force), hyperbolic and trigonometric potentials:

$$
V(\rho \cdot q)=\left\{\begin{array}{cl}
1 /(\rho \cdot q)^{2}, & \text { type I, } \\
a^{2} / \sinh ^{2} a(\rho \cdot q), & \text { type II, } \quad \rho \in \Delta, \\
a^{2} / \sin ^{2} a(\rho \cdot q), & \text { type III, }
\end{array}\right.
$$

in which $a$ is an arbitrary real positive constant, determining the period of the trigonometric potentials. They imply integrability for all of the Calogero-Moser models based on the crystallographic root systems. Those models based on the non-crystallographic root systems, the dihedral group $I_{2}(m), H_{3}$, and $H_{4}$, are integrable only for the rational potential. The rational potential models are also integrable if a confining harmonic potential

$$
\frac{1}{2} \omega^{2} q^{2}, \quad \omega>0, \quad \text { type } \mathrm{V},
$$

is added to the Hamiltonian. Since we will discuss the universal properties and solutions applicable to all the interaction types as well as those for specific interaction potentials, let us 
adopt the conventional nomenclature for them. We call the models with rational, hyperbolic, trigonometric and rational with harmonic force the type I, II, III and V models, respectively. (Type IV models have elliptic potentials which we will not discuss in this paper.)

The Hamiltonian for the quantum Calogero-Moser model can be written in a 'factorised form':

$$
\begin{aligned}
\mathcal{H} & =\frac{1}{2} \sum_{j=1}^{r}\left(p_{j}-i \frac{\partial W}{\partial q_{j}}\right)\left(p_{j}+i \frac{\partial W}{\partial q_{j}}\right) \\
& =\frac{1}{2} \sum_{j=1}^{r}\left(p_{j}^{2}+\left(\frac{\partial W}{\partial q_{j}}\right)^{2}\right)+\frac{1}{2} \sum_{j=1}^{r} \frac{\partial^{2} W}{\partial q_{j}^{2}}, \\
& =\frac{1}{2} p^{2}+\frac{1}{2} \sum_{\rho \in \Delta_{+}} g_{|\rho|}\left(g_{|\rho|}-1\right)|\rho|^{2} V(\rho \cdot q)+\left(\frac{\omega^{2}}{2} q^{2}\right)-\mathcal{E}_{0}
\end{aligned}
$$

It should be noted that the above factorised Hamiltonian (2.7) consists of an operator part $\hat{\mathcal{H}}$, which is the Hamiltonian in the usual definition, and a constant $\mathcal{E}_{0}$ which is the ground state energy to be discussed later:

$$
\begin{aligned}
\mathcal{H} & =\hat{\mathcal{H}}-\mathcal{E}_{0} \\
\hat{\mathcal{H}} & =\frac{1}{2} p^{2}+\frac{1}{2} \sum_{\rho \in \Delta_{+}} g_{|\rho|}\left(g_{|\rho|}-1\right)|\rho|^{2} V(\rho \cdot q)+\left(\frac{\omega^{2}}{2} q^{2}\right) .
\end{aligned}
$$

The real positive coupling constants $g_{|\rho|}$ are defined on orbits of the corresponding Coxeter group, i.e. they are identical for roots in the same orbit. That is, for the simple Lie algebra cases one coupling constant $g_{|\rho|}=g$ for all roots in simply-laced models and two independent coupling constants, $g_{|\rho|}=g_{L}$ for long roots and $g_{|\rho|}=g_{S}$ for short roots in non-simply laced models. For the $I_{2}(m)$ models, there is one coupling if $m$ is odd, and two independent ones if $m$ is even. Let us call them $g_{e}$ for even roots and $g_{o}$ for odd roots. Throughout this paper we consider the coupling constants at generic values. We parametrise the positive roots of $I_{2}(m)$ as

$$
\rho_{j}=(\cos ((j-1) \pi / m), \sin ((j-1) \pi / m)), \quad j=1, \ldots, m .
$$

The $H_{3}$ and $H_{4}$ models have one coupling constant $g_{|\rho|}=g$, since these root systems are simply-laced. It should be noted that the operator part of the Hamiltonian $\hat{\mathcal{H}}$ is strictly positive for $g_{|\rho|} \geq 1$. 
The simplest way to introduce the factorised form is through supersymmetry [9, 23], in which function $W$ is called a superpotential:

$$
W(q)=\sum_{\rho \in \Delta_{+}} g_{|\rho|} \ln |w(\rho \cdot q)|+\left(-\frac{\omega}{2} q^{2}\right), \quad g_{|\rho|}>0, \quad \omega>0 .
$$

The potential $V(u)(2.3)$ and the function $w(u)$ are related by

$$
\begin{aligned}
y(u) & \equiv \frac{d}{d u} x(u), \quad \frac{d w(u)}{d u} / w(u) \equiv x(u), \\
V(u) & =-y(u)=x^{2}(u)+a^{2} \times\left\{\begin{aligned}
0 & \text { rational, } \\
-1 & \text { hyperbolic, } \\
1 & \text { trigonometric. }
\end{aligned}\right.
\end{aligned}
$$

The following Table I gives these functions for each potential:

\begin{tabular}{|lc|c|c|c|}
\hline potential & type & $w(u)$ & $x(u)$ & $y(u)$ \\
\hline rational & I \& V & $u$ & $1 / u$ & $-1 / u^{2}$ \\
\hline hyperbolic & II & $\sinh a u$ & $a \operatorname{coth} a u$ & $-a^{2} / \sinh ^{2} a u$ \\
\hline trigonometric & III & $\sin a u$ & $a \cot a u$ & $-a^{2} / \sin ^{2} a u$ \\
\hline
\end{tabular}

Table I: Functions appearing in the Lax pair and superpotential.

For proofs that the factorised Hamiltonian (2.6) actually gives the quantum Hamiltonian (2.7) for all the root systems and potentials see [5, 11, 9]. It is easy to verify that for any potential $V(u)$, the Hamiltonian is invariant under reflection of the phase space variables in the hyperplane perpendicular to any root

$$
\mathcal{H}\left(s_{\alpha}(p), s_{\alpha}(q)\right)=\mathcal{H}(p, q), \quad \forall \alpha \in \Delta
$$

with $s_{\alpha}$ defined by (A.2).

Some remarks are in order. For all of the root systems and for any choice of potential (2.3), the Calogero-Moser model has a hard repulsive potential $\sim 1 /(\alpha \cdot q)^{2}$ near the reflection hyperplane $H_{\alpha}=\left\{q \in \mathbf{R}^{r}, \alpha \cdot q=0\right\}$. The strength of the singularity is given by the coupling constant $g_{|\alpha|}\left(g_{|\alpha|}-1\right)$ which is independent of the choice of the normalisation of the roots. (Thus for rational models with/without harmonic force there is equivalence, $A_{2} \cong I_{2}(3)$, $B_{2} \cong I_{2}(4), G_{2} \cong I_{2}(6), B_{r} \cong C_{r} \cong B C_{r}$.) This determines the form of the ground state wavefunction, as we will see in subsection 2.2. This repulsive potential is classically insurmountable. Thus the motion is always confined within one Weyl chamber. This feature allows us to constrain the configuration space to the principal Weyl chamber (П: set of simple roots, see Appendix A)

$$
P W=\left\{q \in \mathbf{R}^{r} \mid \alpha \cdot q>0, \quad \alpha \in \Pi\right\},
$$


without loss of generality. In the case of the trigonometric potential, the configuration space is further limited due to the periodicity of the potential to

$$
P W_{T}=\left\{q \in \mathbf{R}^{r} \mid \alpha \cdot q>0, \quad \alpha \in \Pi, \quad \alpha_{h} \cdot q<\pi / a\right\}
$$

where $\alpha_{h}$ is the highest root.

The fact that the classical motions are confined in the Weyl chamber (alcove) $P W\left(P W_{T}\right)$ does not necessarily mean that the corresponding quantum wavefunctions vanish identically outside of the region. On the contrary, as we will see soon, the ground state wavefunction (subsection 2.2) and all the other excited states wavefunctions (section 3) are Coxeter invariant, reflecting the Coxeter invariance of the Hamiltonian (2.14). In the early years of Calogero-Moser models in which those based on the $A_{r}$ root system were mainly discussed, these Coxeter invariant solutions were considered as totally symmetric states of bosonic systems. We will not, however, adopt this interpretation, for in the models based on the other root systems the reflection is not the same as particle interchange. The quantum theory we are discussing is the so-called first quantised theory. That is, the notions of identical particles and the associated statistics are non-existent.

\subsection{Ground state wavefunction and energy}

One merit of the factorised Hamiltonian (2.5) is the ease of derivation of the ground state wavefunction and of the Hamiltonian (3.3) derived by the similarity transformation in terms of the ground state wavefunction. Supersymmetric formulation of the Calogero-Moser models [23, 16, 9] provides a natural setting for the introduction of the factorised Hamiltonian. The universal ground state wavefunction is

$$
\Phi_{0}(q)=e^{W(q)}=\prod_{\rho \in \Delta_{+}}|w(\rho \cdot q)|^{g_{|\rho|} \mid} e^{-\frac{\omega}{2} q^{2}}
$$

The exponential factor $e^{-\frac{\omega}{2} q^{2}}$ exists only for the rational potential case with the harmonic confining force. It is easy to see that it is an eigenstate of the Hamiltonian (2.5) with zero eigenvalue:

$$
\mathcal{H} \Phi_{0}(q)=\frac{1}{2} \sum_{j=1}^{r}\left(p_{j}-i \frac{\partial W}{\partial q_{j}}\right)\left(p_{j}+i \frac{\partial W}{\partial q_{j}}\right) \Phi_{0}(q)=0
$$

since it satisfies

$$
\left(p_{j}+i \frac{\partial W}{\partial q_{j}}\right) e^{W(q)}=0, \quad j=1, \ldots, r
$$


By using the decomposition of the factorised Hamiltonian into the operator Hamiltonian (2.9) and a constant, we obtain

$$
\hat{\mathcal{H}} e^{W} \equiv\left(\frac{1}{2} p^{2}+\frac{1}{2} \sum_{\rho \in \Delta_{+}} g_{|\rho|}\left(g_{|\rho|}-1\right)|\rho|^{2} V(\rho \cdot q)+\left(\frac{\omega^{2}}{2} q^{2}\right)\right) e^{W}=\mathcal{E}_{0} e^{W}
$$

In other words, the above solution (2.17) provides an eigenstate of the Hamiltonian operator $\hat{\mathcal{H}}$ with energy $\mathcal{E}_{0}$. The fact that it is a ground state (for type I, III and V) can be easily shown within the framework of the supersymmetric model [9] thanks to the positivity of the supersymmetric Hamiltonian. It should be stressed that $\mathcal{E}_{0}$ is determined purely algebraically [9], without really applying the operator on the left hand side of (2.20) to the wavefunction. This type of ground states has been known for some time. It is derived by various methods, see for example [3, 5], and also by using supersymmetric quantum mechanics for the models based on classical root systems [23, 16].

The ground state energy for the rational potential cases are

$$
\mathcal{E}_{0}=\left\{\begin{array}{cc}
0 & \text { type I, } \\
\omega\left(\frac{r}{2}+\sum_{\rho \in \Delta_{+}} g_{|\rho|}\right) & \text { type V. }
\end{array}\right.
$$

The same for the hyperbolic and trigonometric potential cases are

$$
\mathcal{E}_{0}=2 a^{2} \varrho^{2} \times\left\{\begin{array}{cl}
-1 & \text { hyperbolic } \\
1 & \text { trigonometric }
\end{array}\right.
$$

in which

$$
\varrho=\frac{1}{2} \sum_{\rho \in \Delta_{+}} g_{|\rho|} \rho
$$

can be considered as a 'deformed Weyl vector' [5, 22]. Again these formulas are universal. That is they apply to all of the Calogero-Moser models based on any root systems. A negative $\mathcal{E}_{0}$ for the obviously positive Hamiltonian of the hyperbolic potential model indicates that the interpretation of $e^{W}$ as an eigenfunction is not correct. This function diverges as $|q| \rightarrow \infty$ for the hyperbolic and the rational potential cases, destroying the hermiticity of the Hamiltonian. Obviously we have

$$
\int_{P W\left(P W_{T}\right)} e^{2 W(q)} d q=\left\{\begin{array}{cl}
\infty & : \text { type I and II } \\
\text { finite } & : \text { type III and V }
\end{array}\right.
$$

in which $P W$ and $P W_{T}$ denote that the integration is over the regions defined in 2.15) and (2.16). Naturally, most existing results in quantum Calogero-Moser models are for the 
models with trigonometric potential and the rational potential with harmonic force which have normalisable states and discrete spectra.

It should be remarked that the domain of the universal ground state wavefunction $\Phi_{0}$ could be considered as the entire $\mathbf{R}^{r}$ space except for the points on the reflection hyperplanes, that is the disjoint union of all the Weyl chambers (alcoves), instead of the initial Weyl chamber/alcove $\left(P W, P W_{T}\right)$ in which the classical motions are restricted due to the singular potential. In fact, $\Phi_{0}$ and $W$ are characterised as Coxeter invariant:

$$
\check{s}_{\rho} \Phi_{0}=\Phi_{0}, \quad \check{s}_{\rho} W=W, \quad \forall \rho \in \Delta
$$

in which $\check{s}_{\rho}$ is the representation of the reflection in the function space. For an arbitrary function $f$ of $q$, its action is defined by

$$
\left(\check{s}_{\rho} f\right)(q)=f\left(s_{\rho}(q)\right) \text {. }
$$

This definition can be generalised to the entire Coxeter group $G_{\Delta}$ : for an arbitrary element $g$ of $G_{\Delta}, \check{g}$ is defined by:

$$
(\check{g} f)(q)=f\left(g^{-1}(q)\right), \quad \forall g \in G_{\Delta}
$$

In the rest of this paper we discuss mainly the type III and V models which have normalisable states and discrete spectra.

\section{Coxeter invariant excited states, triangularity and spectrum}

In this section we show that all the excited states wavefunctions are Coxeter invariant, too. In other words, the Fock space consists of Coxeter invariant functions only. With the knowledge of the ground state wavefunction $e^{W}$, the other states of the Calogero-Moser models can be easily obtained as eigenfunctions of a differential operator $\tilde{\mathcal{H}}$ obtained from $\mathcal{H}$ by a similarity transformation:

$$
\begin{gathered}
\tilde{\mathcal{H}}=e^{-W} \mathcal{H} e^{W} \\
=e^{-W}\left(\frac{1}{2} p^{2}+\frac{1}{2} \sum_{\rho \in \Delta_{+}} g_{|\rho|}\left(g_{|\rho|}-1\right)|\rho|^{2} V(\rho \cdot q)+\left(\frac{\omega^{2}}{2} q^{2}\right)-\mathcal{E}_{0}\right) e^{W}, \\
\tilde{\mathcal{H}} \Psi_{\lambda}=\lambda \Psi_{\lambda} \Longleftrightarrow \mathcal{H} \Psi_{\lambda} e^{W}=\lambda \Psi_{\lambda} e^{W} .
\end{gathered}
$$


Thanks to the factorised form of the Hamiltonian $\mathcal{H}$ (2.5), (2.6), the transformed Hamiltonian $\tilde{\mathcal{H}}$ takes a simple form:

$$
\tilde{\mathcal{H}}=-\frac{1}{2} \sum_{j=1}^{r}\left(\frac{\partial^{2}}{\partial q_{j}^{2}}+2 \frac{\partial W}{\partial q_{j}} \frac{\partial}{\partial q_{j}}\right) .
$$

The Coxeter invariance of $W$ implies those of $\mathcal{H}$ and $\tilde{\mathcal{H}}$ :

$$
\check{s}_{\rho} \mathcal{H} \check{s}_{\rho}=\mathcal{H}, \quad \check{s}_{\rho} \tilde{\mathcal{H}}_{\rho}=\tilde{\mathcal{H}}, \quad \forall \rho \in \Delta .
$$

For type I and III models we introduce proper bases of Fock space consisting of Coxeter invariant functions and show that the above Hamiltonian $\tilde{\mathcal{H}}(\sqrt[3.3)]{)}$ is triangular in these bases. This establishes the integrability of the type I and III models universally 1 and also gives the entire spectrum of the Hamiltonian, see (3.12), (3.13) and (3.44).

\subsection{Rational potential with harmonic force}

First, let us determine the structure of the set of eigenfunctions of the transformed Hamiltonian $\tilde{\mathcal{H}}$, for the type $\mathrm{V}$ models:

$$
\tilde{\mathcal{H}}=\omega q \cdot \frac{\partial}{\partial q}-\frac{1}{2} \sum_{j=1}^{r} \frac{\partial^{2}}{\partial q_{j}^{2}}-\sum_{\rho \in \Delta_{+}} \frac{g_{|\rho|}}{\rho \cdot q} \rho \cdot \frac{\partial}{\partial q} .
$$

Obviously a constant and $\omega q^{2}-\mathcal{E}_{0} / \omega$ are its eigenfunctions with eigenvalue 0 and $2 \omega$, respectively. Let us suppose that a polynomial $P(q)$ is an eigenfunction of $\tilde{\mathcal{H}}$ :

$$
\tilde{\mathcal{H}} P(q)=\lambda P(q)
$$

Due to the Coxeter invariance of $\tilde{\mathcal{H}}(3.4)$, we know that $\check{s}_{\rho} P$ together with the difference

$$
Q=\left(1-\check{s}_{\rho}\right) P
$$

are also eigenfunctions with the same eigenvalue:

$$
\tilde{\mathcal{H}} Q(q)=\lambda Q(q)
$$

if the latter is not identically zero. Since $Q$ is a polynomial which is odd under reflection $\check{s}_{\rho}$

$$
\check{s}_{\rho} Q(q)=-Q(q)
$$

\footnotetext{
${ }^{1}$ Triangularity of the $A_{r}$ type $\mathrm{V}$ and III Hamiltonians was noted in the original papers of Calogero [1] and Sutherland [2]. That of rank two models in the Coxeter invariant bases was shown in [22, 24].
} 
it can be factorised as

$$
Q(q)=(\rho \cdot q)^{2 n+1} \tilde{Q}(q),\left.\quad \tilde{Q}\right|_{\rho \cdot q=0} \neq 0,
$$

with a non-negative integer $n$ and a polynomial $\tilde{Q}$. By substituting (3.8) into (3.7) and using the explicit form of $\tilde{\mathcal{H}}$ near the reflection hyperplane $\rho \cdot q=0$, we obtain

$$
-(\rho \cdot q)^{2 n-1}(2 n+1)\left(n+g_{|\rho|}\right) \rho^{2} \tilde{Q}+\mathcal{O}\left[(\rho \cdot q)^{2 n}\right]=\lambda(\rho \cdot q)^{2 n+1} \tilde{Q},
$$

which would imply the vanishing of $\tilde{Q}$ on the reflection hyperplane

$$
\left.\tilde{Q}\right|_{\rho \cdot q=0}=0,
$$

an obvious contradiction. Thus we are led to the conclusion that the eigenfunctions are Coxeter invariant polynomials and that the Hamiltonian $\mathcal{H}$ (3.5) maps a Coxeter invariant polynomial to another.

An obvious basis in the space of Coxeter invariant polynomials is the homogeneous polynomials of various degrees. This basis has a natural order given by the degree. For a given degree the space of homogeneous Coxeter invariant polynomials is finite-dimensional. The explicit form of $\tilde{\mathcal{H}}(3.5)$ shows that it is lower triangular in this basis and the diagonal elements are $\omega \times$ degree as given by the first term. Independent Coxeter invariant polynomials exist at the degrees $f_{j}$ listed in Table II:

$$
f_{j}=1+e_{j}, \quad j=1, \ldots, r
$$

in which $\left\{e_{j}\right\}, j=1, \ldots, r$, are the exponents of $\Delta$. Let us denote them by

$$
z_{1}(q), \ldots, z_{r}(q) ; \quad z_{j}(\kappa q)=\kappa^{f_{j}} z_{j}(q) .
$$

\begin{tabular}{||c|l||c|l||}
\hline$\Delta$ & $f_{j}=1+e_{j}$ & $\Delta$ & $f_{j}=1+e_{j}$ \\
\hline$A_{r}$ & $2,3,4, \ldots, r+1$ & $E_{8}$ & $2,8,12,14,18,20,24,30$ \\
\hline$B_{r}$ & $2,4,6, \ldots, 2 r$ & $F_{4}$ & $2,6,8,12$ \\
\hline$C_{r}$ & $2,4,6, \ldots, 2 r$ & $G_{2}$ & 2,6 \\
\hline$D_{r}$ & $2,4, \ldots, 2 r-2 ; r$ & $I_{2}(m)$ & $2, m$ \\
\hline$E_{6}$ & $2,5,6,8,9,12$ & $H_{3}$ & $2,6,10$ \\
\hline$E_{7}$ & $2,6,8,10,12,14,18$ & $H_{4}$ & $2,12,20,30$ \\
\hline
\end{tabular}

Table II: The degrees $f_{j}$ in which independent Coxeter invariant polynomials exist.

Thus we arrive at:

the quantum Calogero-Moser models with the rational potential and the harmonic confining 
force is algebraically solvable for any (crystallographic and non-crystallographic) root system $\Delta$. The spectrum of the operator Hamiltonian $\hat{\mathcal{H}}$ is

$$
\omega N+\mathcal{E}_{0}
$$

with a non-negative integer $N$ which can be expressed as

$$
N=\sum_{j=1}^{r} n_{j} f_{j}, \quad n_{j} \in \mathbf{Z}_{+},
$$

and the degeneracy of the above eigenvalue (3.12) is the number of different solutions of (3.13) for given $N$. This is generalisation of Calogero's original argument for the $A_{r}$ model [1] to the models based on arbitrary root systems. Now let us denote by $\vec{N}$ the set of non-negative integers in (3.13):

$$
\vec{N}=\left(n_{1}, n_{2}, \ldots, n_{r}\right)
$$

and by $\phi_{\vec{N}}(q)$ the homogeneous Coxeter invariant polynomial of determined by $\vec{N}$ and the above basis $\left\{z_{j}\right\}$ (3.11):

$$
\phi_{\vec{N}}(q)=\prod_{j=1}^{r} z_{j}^{n_{j}}(q) .
$$

As shown above, there exists a unique eigenstate $\psi_{\vec{N}}(q)$ for each $\phi_{\vec{N}}(q)$ :

$$
\begin{aligned}
\psi_{\vec{N}}(q) & =\phi_{\vec{N}}(q)+\sum_{\overrightarrow{N^{\prime}}<\vec{N}} d_{\vec{N}^{\prime}} \phi_{\vec{N}^{\prime}}(q), \quad d_{\vec{N}^{\prime}}: \text { const } \\
\tilde{\mathcal{H}} \psi_{\vec{N}}(q) & =\omega N \psi_{\vec{N}}(q) .
\end{aligned}
$$

It satisfies the orthogonality relation

$$
\left(\psi_{\vec{N}}, \phi_{\vec{N}^{\prime}}\right)=0, \quad \vec{N}^{\prime}<\vec{N}
$$

with respect to the inner product in $P W$ :

$$
(\psi, \varphi)=\int_{P W} \psi^{*}(q) \varphi(q) e^{2 W(q)} d q
$$

These polynomials $\left\{\psi_{\vec{N}}(q)\right\}$ are generalisations of the multivariable Laguerre (Hermite) polynomials [14] known for the $A_{r}\left(B_{r}, D_{r}\right)$ root systems to arbitrary root systems.

Some remarks are in order. 
1. There is no Coxeter invariant linear function in $q$. The quadratic invariant polynomial $q^{2}=q \cdot q$ exists in all the root systems. This corresponds to the universal fact that $f_{1}=2,\left(e_{1}=1\right)$ for all the root systems. Moreover, this is related to the fact that a special sub-series of the excited states with $N=2 n_{1}=n_{1} f_{1}, n_{j}=0, j \geq 2$, can be expressed universally in terms of Laguerre polynomials in $q^{2}$. This will be discussed at the end of section 5 and in subsection 7.2 .

2. The other Coxeter invariants corresponding to the degrees $f_{2}, \ldots, f_{r}$ could be interpreted as special 'angular' variables of a unit sphere $S^{r-1}\left(q^{2}=1\right)$, with the first Coxeter invariant $\sqrt{q^{2}}$ being the radial coordinate. These would provide proper variables for describing solutions. Solutions in terms of separation of variables are in general possible only for the simplest cases, that is the rank two models, which will be demonstrated in subsection 7.3 .

3. For $\Delta=A_{1}$, the simplest root system of rank one, the Hamiltonian $\tilde{\mathcal{H}}$ can be rewritten in terms of a Coxeter invariant variable $u=\omega q^{2}$ as:

$$
\tilde{\mathcal{H}}=\omega q \frac{d}{d q}-\frac{1}{2} \frac{d^{2}}{d q^{2}}-\frac{g}{q} \frac{d}{d q}=-2 \omega\left\{u \frac{d^{2}}{d u^{2}}+\left(g+\frac{1}{2}-u\right) \frac{d}{d u}\right\} .
$$

The Laguerre polynomial satisfying the differential equation

$$
\left\{u \frac{d^{2}}{d u^{2}}+\left(g+\frac{1}{2}-u\right) \frac{d}{d u}+n\right\} L_{n}^{\left(g-\frac{1}{2}\right)}(u)=0,
$$

provides an eigenfunction with eigenvalue $2 \omega n$, which corresponds to the eigenvalue $2 \omega n+\mathcal{E}_{0}$ of $\hat{\mathcal{H}}$. This is a well-known result.

4. Triangularity of the type I models is also obvious from the above argument.

\subsection{Trigonometric potential}

Here we consider those root systems associated with Lie algebras. In order to determine the excited states of the type III models, we have to consider the periodicity. The superpotential $W$ and the Hamiltonian $\mathcal{H}$ are invariant under the following translation:

$$
W\left(q^{\prime}\right)=W(q), \quad \mathcal{H}\left(p, q^{\prime}\right)=\mathcal{H}(p, q), \quad q^{\prime}=q+l^{\vee} \pi / a
$$

in which $l^{\vee}$ is an element of the dual weight lattice, that is

$$
l^{\vee}=\sum_{j=1}^{r} l_{j} \frac{2}{\alpha_{j}^{2}} \lambda_{j}, \quad l_{j} \in \mathbf{Z}, \quad \alpha_{j} \in \Pi, \quad \alpha_{j}^{\vee} \cdot \lambda_{k}=\delta_{j k} .
$$


As is well known in quantum mechanics with periodic potentials, the wavefunctions diagonalising the translation operators are expressed as

$$
e^{2 i a \mu \cdot q}\left(\sum_{\alpha \in L(\Delta)} b_{\alpha} e^{2 i a \alpha \cdot q}\right) e^{W}, \quad b_{\alpha}: \text { const }, \quad L(\Delta) \text { : root lattice, }
$$

in which a vector $\mu \in \mathbf{R}^{r}$ is as yet unspecified. In other words, up to the overall phase factor $e^{2 i a \mu \cdot q}$, this is a Fourier expansion in terms of the simple roots.

Let $P_{T}(q)$ be a polynomial in $e^{ \pm 2 i a \alpha_{j} \cdot q}, \alpha_{j} \in \Pi$ and suppose that a function $\phi(q)$

$$
\phi(q)=e^{2 i a \mu \cdot q} P_{T}(q), \quad \mu \in \mathbf{R}^{r}
$$

is an eigenfunction of $\tilde{\mathcal{H}}$ :

$$
\tilde{\mathcal{H}} \phi(q)=\lambda \phi(q)
$$

in which the explicit form of $\tilde{\mathcal{H}}$ is given by

$$
\tilde{\mathcal{H}}=-\frac{1}{2} \sum_{j=1}^{r} \frac{\partial^{2}}{\partial q_{j}^{2}}-a \sum_{\rho \in \Delta_{+}} g_{|\rho|} \cot (a \rho \cdot q) \rho \cdot \frac{\partial}{\partial q} .
$$

As above, due to the Coxeter invariance of $\tilde{\mathcal{H}}(3.4)$, we know that $\check{s}_{\rho} \phi$ together with the difference

$$
\varphi=\left(1-\check{s}_{\rho}\right) \phi
$$

are also eigenfunctions with the same eigenvalue:

$$
\tilde{\mathcal{H}} \varphi(q)=\lambda \varphi(q)
$$

Since $\varphi$ is odd under reflection $\check{s}_{\rho}$

$$
\check{s}_{\rho} \varphi(q)=-\varphi(q)
$$

it can be expressed as

$$
\varphi(q)=(\rho \cdot q)^{2 n+1} \tilde{\varphi}(q)+\mathcal{O}\left[(\rho \cdot q)^{2 n+3}\right],\left.\quad \tilde{\varphi}\right|_{\rho \cdot q=0} \neq 0
$$

in a neighbourhood of the reflection hyperplane $\rho \cdot q=0$. In this neighbourhood, the singularity structure of $\tilde{\mathcal{H}}$ for the trigonometric potential is the same as that of $\tilde{\mathcal{H}}$ for the rational potential discussed in the previous subsection. Thus we obtain, as before, a contradiction $\left.\tilde{\varphi}\right|_{\rho \cdot q=0}=0$. In other words, the eigenfunction $\phi(3.25)$ must be Coxeter invariant. This in turn requires that the unspecified vector $\mu$ in (3.25) to be an element of the weight lattice

$$
\mu \in \Lambda(\Delta)
$$


Since

$$
\check{s}_{\rho} \phi(q)=e^{2 i a s_{\rho}(\mu) \cdot q} \check{s}_{\rho} P_{T}(q)=e^{2 i a\left(\mu \cdot q-\rho^{\vee} \cdot \mu \rho \cdot q\right)} \breve{s}_{\rho} P_{T}(q),
$$

the following condition is necessary, but not sufficient,

$$
\rho^{\vee} \cdot \mu \in \mathbf{Z}, \quad \forall \rho \in \Delta
$$

for the Coxeter invariance of $\phi$. Thus we arrive at (3.30).

Let us introduce a basis for the Coxeter invariant functions of the form (3.25). Let $\lambda$ be a dominant weight

$$
\lambda=\sum_{j=1}^{r} m_{j} \lambda_{j}, \quad m_{j} \in \mathbf{Z}_{+},
$$

and $W_{\lambda}$ be the orbit of $\lambda$ by the action of the Weyl group:

$$
W_{\lambda}=\left\{\mu \in \Lambda(\Delta) \mid \quad \mu=g(\lambda), \quad \forall g \in G_{\Delta}\right\} .
$$

We define

$$
\phi_{\lambda}(q) \equiv \sum_{\mu \in W_{\lambda}} e^{2 i a \mu \cdot q}
$$

which is Coxeter invariant. The set of functions $\left\{\phi_{\lambda}\right\}$ has an order $>$ :

$$
|\lambda|^{2}>\left|\lambda^{\prime}\right|^{2} \Rightarrow \phi_{\lambda}>\phi_{\lambda^{\prime}}
$$

Next we show that $\tilde{\mathcal{H}}$ is lower triangular in this basis. By using (3.27) we obtain

$$
\tilde{\mathcal{H}} \phi_{\lambda}=2 a^{2} \lambda^{2} \phi_{\lambda}-2 i a^{2} \sum_{\rho \in \Delta_{+}} \sum_{\mu \in W_{\lambda}} g_{|\rho|} \cot (a \rho \cdot q)(\rho \cdot \mu) e^{2 i a \mu \cdot q} .
$$

First let us fix one positive root $\rho$ and a weight $\mu$ in $W_{\lambda}$ such that $\rho \cdot \mu \neq 0$. Then

$$
\mu^{\prime} \equiv s_{\rho}(\mu)=\mu-\left(\rho^{\vee} \cdot \mu\right) \rho \in W_{\lambda}, \quad \rho \cdot \mu^{\prime}=-\rho \cdot \mu .
$$

Without loss of generality we may assume

$$
\rho^{\vee} \cdot \mu=k>0, \quad k \in \mathbf{Z} .
$$

The contribution of the pair $\left(\mu, \mu^{\prime}\right)$ in the summation of (3.36) reads

$$
\begin{aligned}
& |\rho \cdot \mu| e^{2 a i \mu \cdot q}\left(1-e^{-2 a i k \rho \cdot q}\right) \cot (a \rho \cdot q) \\
= & i|\rho \cdot \mu|\left(e^{2 a i \mu \cdot q}+e^{2 a i \mu^{\prime} \cdot q}+2 \sum_{j=1}^{k-1} e^{2 a i(\mu-j \rho) \cdot q}\right),
\end{aligned}
$$


which is the generalisation of Sutherland's fundamental identity eq(15) in [2] to arbitrary root systems. The summation in the expression correspond to $\phi_{\lambda^{\prime}}$ with $\lambda^{\prime}$ being lower than $\lambda$. Thus (3.36) reads

$$
\tilde{\mathcal{H}} \phi_{\lambda}=2 a^{2} \lambda^{2} \phi_{\lambda}+2 a^{2} \sum_{\rho \in \Delta_{+}} \sum_{\mu \in W_{\lambda}} g_{|\rho|}|\rho \cdot \mu| e^{2 i a \mu \cdot q}+\sum_{\left|\lambda^{\prime}\right|<|\lambda|} c_{\lambda^{\prime}} \phi_{\lambda^{\prime}}
$$

in which $\left\{c_{\lambda^{\prime}}\right\}$ 's are constants. It is easy to see that $\left(\mu=g(\lambda), \exists g \in G_{\Delta}\right)$

$$
\sum_{\rho \in \Delta_{+}} g_{|\rho|}|\rho \cdot \mu|=\sum_{\rho \in \Delta_{+}} g_{|\rho|}|g(\rho) \cdot \lambda|=\left(\sum_{\rho \in \Delta_{+}} g_{|\rho|} \rho\right) \cdot \lambda=2 \varrho \cdot \lambda
$$

which is independent of $\mu$. Thus we have demonstrated the triangularity of $\tilde{\mathcal{H}}$ :

$$
\tilde{\mathcal{H}} \phi_{\lambda}=2 a^{2}\left(\lambda^{2}+2 \varrho \cdot \lambda\right) \phi_{\lambda}+\sum_{\left|\lambda^{\prime}\right|<|\lambda|} c_{\lambda^{\prime}} \phi_{\lambda^{\prime}}
$$

or that of $\hat{\mathcal{H}}$

$$
\hat{\mathcal{H}} \phi_{\lambda} e^{W}=2 a^{2}(\lambda+\varrho)^{2} \phi_{\lambda} e^{W}+\sum_{\left|\lambda^{\prime}\right|<|\lambda|} c_{\lambda^{\prime}} \phi_{\lambda^{\prime}} e^{W}
$$

with the eigenvalue

$$
2 a^{2}(\lambda+\varrho)^{2}
$$

In other words, for each dominant weight $\lambda$ there exists an eigenstate of $\tilde{\mathcal{H}}$ with eigenvalue proportional to $\lambda(\lambda+2 \varrho)$. Let us denote this eigenfunction by $\psi_{\lambda}(q)$ :

$$
\begin{aligned}
\psi_{\lambda}(q) & =\phi_{\lambda}(q)+\sum_{|\lambda|^{\prime}<|\lambda|} d_{\lambda^{\prime}} \phi_{\lambda^{\prime}}(q), \quad d_{\lambda^{\prime}}: \text { const }, \\
\tilde{\mathcal{H}} \psi_{\lambda}(q) & =2 a^{2} \lambda(\lambda+2 \varrho) \psi_{\lambda}(q)
\end{aligned}
$$

and call it a generalised Jack polynomial [25]-28]. It satisfies the orthogonality relation

$$
\left(\psi_{\lambda}, \phi_{\lambda^{\prime}}\right)=0, \quad|\lambda|^{\prime}<|\lambda|
$$

with respect to the inner product in $P W_{T}$ :

$$
(\psi, \varphi)=\int_{P W_{T}} \psi^{*}(q) \varphi(q) e^{2 W(q)} d q
$$

In the $A_{r}$ model, specifying a dominant weight $\lambda$ is the same as giving a Young diagram which designates a Jack polynomial. It should be emphasised, however, that $\left\{\psi_{\lambda}\right\}$ are not identical to the Jack polynomials even for the $A_{r}$ root systems, because of different treatments of the 
center of mass coordinates. Detailed properties of these polynomials for various root systems will be published elsewhere.

Thus we arrive at:

the quantum Calogero-Moser models with the trigonometric potential are algebraically solvable for any crystallographic root system $\Delta$. The spectrum of the Hamiltonian $\hat{\mathcal{H}}$ is given by (3.44) in which $\lambda$ is an arbitrary dominant weight. This is generalisation of Sutherland's original argument [2] to the models based on arbitrary root systems.

Some remarks are in order.

1. The weights $\mu$ appearing in the lower order terms $\left\{\phi_{\lambda^{\prime}}\right\}$ 's are those weights contained in the Lie algebra representation belonging to the highest weight $\lambda$.

2. As a simple corollary we find that for a minimal weight $\lambda$,

$$
\psi_{\lambda}(q)=\phi_{\lambda}(q)=\sum_{\mu \in W_{\lambda}} e^{2 i a \mu \cdot q}
$$

is an eigenfunction of $\tilde{\mathcal{H}}$. A minimal representation [6] consists of a single Weyl orbit and all of its weights $\mu$ satisfy $\rho^{\vee} \cdot \mu=0, \pm 1, \forall \rho \in \Delta$.

3. If $\lambda=\alpha_{h}$, the highest root of a simply-laced root system, $W_{\lambda}$ is the set of roots itself. Then the lower order terms are constants only. We find that

$$
\psi_{\alpha_{h}}(q)=2 \sum_{\rho \in \Delta_{+}}\left(\cos (2 a \rho \cdot q)+g \rho^{2} / \alpha_{h} \cdot\left(\alpha_{h}+2 \varrho\right)\right)
$$

is an eigenstate of $\tilde{\mathcal{H}}$.

4. If $\lambda=\alpha_{S h}$, the highest short root of a non-simply laced root system, $W_{\lambda}$ is the set of short roots itself. The lower order terms are constants, too. Similarly as above, we find that

$$
\psi_{\alpha_{S h}}(q)=2 \sum_{\rho \in \Delta_{L+}} \cos (2 a \rho \cdot q)+2 \sum_{\rho \in \Delta_{S+}}\left(\cos (2 a \rho \cdot q)+g_{S} \rho_{S}^{2} / \alpha_{S h} \cdot\left(\alpha_{S h}+2 \varrho\right)\right)
$$

is an eigenstate of $\tilde{\mathcal{H}}$. Here $\Delta_{L(S)}$ is the set of long (short) roots.

5. If $-\lambda \notin W_{\lambda}$ then there is another set of functions containing the weight $-\lambda$ which belongs to the same eigenvalue. 
6. The Coxeter invariant trigonometric polynomials specified by the fundamental weights $\left\{\lambda_{j}\right\}$

$$
\phi_{\lambda_{j}}(q)=\sum_{\mu \in W_{\lambda_{j}}} e^{2 i a \mu \cdot q}, \quad \lambda_{j}: \text { fundamental weight }, \quad j=1, \ldots, r
$$

are expected to play the role of the fundamental variables [22, 24].

7. Let us consider the well-known case $\Delta=A_{1}$, the simplest root system of rank one. By rewriting the Hamiltonian $\tilde{\mathcal{H}}$ in terms of the Coxeter invariant variable $z=\cos (a \rho q)$, we obtain

$$
\hat{\mathcal{H}}=-\frac{1}{2} \frac{d^{2}}{d q^{2}}-a g \rho \cot (a \rho q) \frac{d}{d q}=-\frac{1}{2} a^{2}|\rho|^{2}\left\{\left(1-z^{2}\right) \frac{d^{2}}{d z^{2}}-(1+2 g) z \frac{d}{d z}\right\} .
$$

The Gegenbauer polynomials [5], a special case of Jacobi polynomials $P_{n}^{(\alpha, \beta)}$ provide eigenfunctions:

$$
P_{n}^{\left(g-\frac{1}{2}, g-\frac{1}{2}\right)}(\cos (a \rho q)), \quad \mathcal{E}=a^{2}|\rho|^{2}(n+g)^{2} / 2, \quad n \in \mathbf{Z}_{+} .
$$

The Jacobi polynomial $P_{\ell}^{(\alpha, \beta)}(z)$ satisfies differential equation

$$
\left\{\left(1-z^{2}\right) \frac{d^{2}}{d z^{2}}+\beta-\alpha-(2+\alpha+\beta) z \frac{d}{d z}+\ell(\ell+\alpha+\beta+1)\right\} P_{\ell}^{(\alpha, \beta)}(z)=0 .
$$

Here we follow the notation of [29]. They form orthogonal polynomials with weight $e^{2 W}=|\sin (a \rho q)|^{2 g}$ in the interval $q \in[0, \pi / a \rho]$, (2.16). The Gegenbauer polynomials have a definite parity, $(-1)^{n}$, reflecting the periodicity. The fundamental period is $\pi / a \rho$, the length of the interval itself. The (odd) even degree ones corresponding to (half-odd) integer spin representations are (anti-) periodic.

8. Triangularity of type II models follows from the same algebraic reasoning.

\section{Quantum Lax pair and quantum conserved quanti- ties}

Historically, Lax pairs for Calogero-Moser models were presented in terms of Lie algebra representations [3, 5], in particular, the vector representation of the $A_{r}$ models. However, the invariance of Calogero-Moser models is that of Coxeter group but not that of the associated Lie algebra, which does not exist for the non-crystallographic root systems. Thus the universal and Coxeter covariant Lax pairs are given in terms of the representations of the Coxeter group. 


\subsection{General case}

Here we recapitulate the essence of the quantum Lax pair operators for the Calogero-Moser models with degenerate potentials and without spectral parameter. The case with spectral parameter will be discussed briefly in subsection 8.3 in connection with the proof of involution of the quantum conserved quantities for $A_{r}$ model [15]. The quantum Lax pair in this subsection applies to all the degenerate potential cases except for the case of the rational potential with the harmonic force, which will be treated separately in subsection 4.2. For details and a full exposition, see [9]. The Lax operators without spectral parameter are

$$
\begin{aligned}
L(p, q) & =p \cdot \hat{H}+X(q), \quad X(q)=i \sum_{\rho \in \Delta_{+}} g_{|\rho|}(\rho \cdot \hat{H}) x(\rho \cdot q) \hat{s}_{\rho}, \\
M(q) & =\frac{i}{2} \sum_{\rho \in \Delta_{+}} g_{|\rho|}|\rho|^{2} y(\rho \cdot q) \hat{s}_{\rho}-\frac{i}{2} \sum_{\rho \in \Delta_{+}} g_{|\rho|}|\rho|^{2} y(\rho \cdot q) \times I,
\end{aligned}
$$

in which $I$ is the identity operator and $\left\{\hat{s}_{\alpha}, \alpha \in \Delta\right\}$ are the reflection operators of the root system. In contrast with $\left\{\check{s}_{\alpha}\right\}$ operators (2.26) which act in function space, $\left\{\hat{s}_{\alpha}\right\}$ act on a set of $\mathbf{R}^{r}$ vectors $\mathcal{R}=\left\{\mu^{(k)} \in \mathbf{R}^{r}, k=1, \ldots, d\right\}$, permuting them under the action of the reflection group. The vectors in $\mathcal{R}$ form a basis for the representation space $\mathbf{V}$ of dimension d. The operator $M$ satisfies the relation

$$
\sum_{\mu \in \mathcal{R}} M_{\mu \nu}=\sum_{\nu \in \mathcal{R}} M_{\mu \nu}=0
$$

which is essential for deriving quantum conserved quantities. The matrix elements of the operators $\left\{\hat{s}_{\alpha}, \alpha \in \Delta\right\}$ and $\left\{\hat{H}_{j}, j=1, \ldots, r\right\}$ are defined as follows:

$$
\left(\hat{s}_{\rho}\right)_{\mu \nu}=\delta_{\mu, s_{\rho}(\nu)}=\delta_{\nu, s_{\rho}(\mu)}, \quad\left(\hat{H}_{j}\right)_{\mu \nu}=\mu_{j} \delta_{\mu \nu}, \quad \rho \in \Delta, \quad \mu, \nu \in \mathcal{R} .
$$

The form of the function $x$ depends on the chosen potential, and the function $y$ are defined by (2.12), (2.13). Note that these relations are only valid for the degenerate potentials (2.3).

The underlying idea of the Lax operator $L$, (4.1), is quite simple. As seen from (4.9), $L$ is a "square root" of the Hamiltonian. Thus one part of $L$ contains $p$ which is not associated with roots and another part contains $x(\rho \cdot q)$, a "square root" of the potential $V(\rho \cdot q)$, which being associated with a root $\rho$ is therefore accompanied by the reflection operator $\hat{s}_{\rho}$. Another explanation is the factorised Hamiltonian $\mathcal{H}$ (2.5). We obtain, roughly speaking,

$L \sim \sqrt{\mathcal{H}} \sim p+i \frac{\partial W}{\partial q} \hat{s}$ and the property of reflection $\hat{s}^{2}=1$ explains the sign change in the first term in (2.5). 
It is straightforward to show that the quantum Lax equation

$$
\frac{d}{d t} L=i[\mathcal{H}, L]=[L, M]
$$

is equivalent to the quantum equations of motion derived from the Hamiltonian (2.7). From this it follows:

$$
\frac{d}{d t}\left(L^{n}\right)_{\mu \nu}=i\left[\mathcal{H},\left(L^{n}\right)_{\mu \nu}\right]=\left[L^{n}, M\right]_{\mu \nu}=\sum_{\lambda \in \mathcal{R}}\left(\left(L^{n}\right)_{\mu \lambda} M_{\lambda \nu}-M_{\mu \lambda}\left(L^{n}\right)_{\lambda \nu}\right), \quad n=1, \ldots
$$

Thanks to the property of the $M$ operator (4.3):

$$
\sum_{\mu \in \mathcal{R}} M_{\mu \nu}=\sum_{\nu \in \mathcal{R}} M_{\mu \nu}=0
$$

we obtain quantum conserved quantities as the total sum (Ts) of all the matrix elements of $L^{n}$ 円:

$$
Q_{n}=\operatorname{Ts}\left(L^{n}\right) \equiv \sum_{\mu, \nu \in \mathcal{R}}\left(L^{n}\right)_{\mu \nu}, \quad\left[\mathcal{H}, Q_{n}\right]=0, \quad n=1, \ldots
$$

Independent conserved quantities appear at such power $n$ that

$$
n=1+\text { exponent }
$$

of each root system. These are the degrees at which independent Coxeter invariant polynomials exist. There are $r$ exponents for each root system $\Delta$ of rank $r$. Thus we have $r$ independent conserved quantities in Calogero-Moser models. We list in Table II these powers for each root system. In particular, the power 2 is universal to all the root systems and the quantum Hamiltonian (2.7) is given by

$$
\mathcal{H}=\frac{1}{2 C_{\mathcal{R}}} \operatorname{Ts}\left(L^{2}\right)+\text { const }
$$

where the constant $C_{\mathcal{R}}$ is the quadratic Casimir invariant, which depends on the representation. It is defined by

$$
\operatorname{Tr}\left(\hat{H}_{j} \hat{H}_{k}\right) \equiv \sum_{\mu \in \mathcal{R}}\left(\hat{H}_{j} \hat{H}_{k}\right)_{\mu \mu}=\sum_{\mu \in \mathcal{R}} \mu_{j} \mu_{k}=C_{\mathcal{R}} \delta_{j k}
$$

Some remarks are in order.

\footnotetext{
${ }^{2}$ This type of conserved quantities is known for $A_{r}$ models 16, 18.
} 
1. The Lax pair is Coxeter covariant:

$$
L\left(s_{\rho}(p), s_{\rho}(q)\right)_{\mu \nu}=L(p, q)_{\mu^{\prime} \nu^{\prime}}, M\left(s_{\rho}(q)\right)_{\mu \nu}=M(q)_{\mu^{\prime} \nu^{\prime}}, \quad \mu^{\prime} \equiv s_{\rho}(\mu), \nu^{\prime} \equiv s_{\rho}(\nu)
$$

which ensures the Coxeter invariance of the conserved quantities.

2. Lax pairs can be written down in various representations and the quantum conserved quantities $Q_{n}$ do depend on the representations, in general. If necessary, we denote by $Q_{n}^{\mathcal{R}}$ the explicit representation dependence.

3. The availability of plural representations of the Lax pair and the conserved quantities is essential for the completeness of the set of conserved quantities as polynomials of the momentum operators. For example, let us consider the case of $D_{r}$ with even $r$, which has two independent conserved quantities at power $r$, see Table II. At least two different representations of the Lax pair are necessary in order to represent them in the form of (4.7). Those based on the vector, and the (anti)-spinor representations give two independent conserved quantities. For $D_{4}$ case, we obtain

$$
Q_{4}^{v}=2 \sum_{j=1}^{4} p_{j}^{4}, \quad Q_{4}^{s}-Q_{4}^{a}=24 \prod_{j=1}^{4} p_{j},
$$

in which $v, s$ and $a$ stand for the vector, spinor and anti-spinor representations and we have set $g=0$ for simplicity. If two conserved quantities are independent for zero coupling constants, surely they are so at non-vanishing couplings. Here we have used an explicit parametrisation of the $D_{r}$ root system:

$$
D_{r} \text { root system : } \Delta=\left\{ \pm e_{j} \pm e_{k}, \quad j, k=1, \ldots, r \mid e_{j} \in \mathbf{R}^{r}, e_{j} \cdot e_{k}=\delta_{j k}\right\}
$$

4. If a representation $\mathcal{R}$ contains a vector $\mu$ and its negative $-\mu$ at the same time, then we have $\operatorname{Ts}\left(L^{\text {odd }}\right)=0$. In such a case the corresponding Lie algebra representations are called real. In order to construct the odd power conserved quantities appearing in $A_{r}$ for all $r, D_{r}$ for odd $r, E_{6}$ and $I(m)$ for odd $m$, we need a Lax pair in nonreal representations. For $A_{r}$ all the fundamental representations corresponding to the fundamental weights $\lambda_{j}, j=1, \ldots, r$ except for the middle one $\lambda_{(r+1) / 2}$ for odd $r$ are non-real. For $D_{r}$ with odd $r$, the spinor and anti-spinor representations are non-real. For $E_{6}$ the $\mathbf{2 7}$ and $\overline{\mathbf{2 7}}$ are non-real. $I_{2}(m)$ is the symmetry group of a regular $m$-sided 
polygon. The set of $m$ vectors with 'half" angles of the roots (see (2.10)) to be denoted by $V_{m}(\mathrm{~B} .3)$, provides a non-real representation when $m$ is odd.

5. In Appendix B we list for each root system how the full set of independent conserved quantities are obtained by choosing proper representations of the Lax pair.

\subsection{Rational potential with harmonic force}

The quantum Lax pair for the type $\mathrm{V}$ models needs a separate formulation. The explicit form of the Hamiltonian is

$$
\mathcal{H}=\frac{1}{2} p^{2}+\frac{1}{2} \omega^{2} q^{2}+\frac{1}{2} \sum_{\rho \in \Delta_{+}} g_{|\rho|}\left(g_{|\rho|}-1\right) \frac{|\rho|^{2}}{(\rho \cdot q)^{2}}-\mathcal{E}_{0} .
$$

The canonical equations of motion are equivalent to the following Lax equations for $L^{ \pm}$:

$$
\frac{d}{d t} L^{ \pm}=i\left[\mathcal{H}, L^{ \pm}\right]=\left[L^{ \pm}, M\right] \pm i \omega L^{ \pm},
$$

in which (see section 4 of [7) $M$ is the same as before (4.2), and $L^{ \pm}$and $Q$ are defined by

$$
L^{ \pm}=L \pm i \omega Q, \quad Q=q \cdot \hat{H},
$$

with $L, \hat{H}$ as earlier (4.1), (4.4). If we define hermitian operators $\mathcal{L}_{1}$ and $\mathcal{L}_{2}$ by

$$
\mathcal{L}_{1}=L^{+} L^{-}, \quad \mathcal{L}_{2}=L^{-} L^{+},
$$

they satisfy Lax-like equations

$$
\dot{\mathcal{L}}_{k}=\left[\mathcal{L}_{k}, M\right], \quad k=1,2 .
$$

From these we can construct conserved quantities

$$
\operatorname{Ts}\left(\mathcal{L}_{j}^{n}\right), \quad j=1,2, \quad n=1,2, \ldots,
$$

as before. Such quantum conserved quantities have been previously reported for models based on $A_{r}$ root systems [16, 18]. It should be remarked that $\operatorname{Ts}\left(\mathcal{L}_{2}^{n}\right)$ is no longer the same as $\operatorname{Ts}\left(\mathcal{L}_{1}^{n}\right)$ due to quantum corrections. It is elementary to check that the first conserved quantities give the Hamiltonian (4.14)

$$
\mathcal{H} \propto \operatorname{Ts}\left(\mathcal{L}_{1}\right)=\operatorname{Ts}\left(\mathcal{L}_{2}\right)+\text { const }
$$

This then completes the presentation of the quantum Lax pairs and quantum conserved quantities for all of the quantum Calogero-Moser models with non-elliptic potentials. 


\section{Algebraic construction of excited states I}

In this section we show that all the excited states of the type V Calogero-Moser models can be constructed algebraically. Later in section 7 we show the same results in terms of the $\ell$ operators to be introduced in section 6. The main result is surprisingly simple and can be stated universally:

Corresponding to each partition of an integer $N$ which specify the energy level (3.12) into the sum of the degrees of Coxeter invariant polynomials (3.13), we have an eigenstate of the Hamiltonian $\hat{\mathcal{H}}$ with eigenvalue $\omega N+\mathcal{E}_{0}$ :

$$
\prod_{j=1}^{r}\left(B_{f_{j}}^{+}\right)^{n_{j}} e^{W}, \quad N=\sum_{j=1}^{r} n_{j} f_{j}, \quad n_{j} \in \mathbf{Z}_{+},
$$

in which the integers $\left\{f_{j}\right\}, j=1, \ldots, r$ are listed in Table II. They exhaust all the excited states. In other words the above states give the complete basis of the Fock space. The creation operators $B_{f_{j}}^{+}$and the corresponding annihilation operators $B_{f_{j}}^{-}$are defined in terms of the Lax operators $L^{ \pm}(4.16)$ as follows:

$$
B_{f_{j}}^{ \pm}=\operatorname{Ts}\left(L^{ \pm}\right)^{f_{j}}, \quad j=1, \ldots, r
$$

They are hermitian conjugate to each other

$$
\left(B_{f_{j}}^{ \pm}\right)^{\dagger}=B_{f_{j}}^{\mp}
$$

with respect to the standard hermitian inner product of the states defined in $P W$ :

$$
(\psi, \varphi)=\int_{P W} \psi^{*}(q) \varphi(q) d q
$$

We will show later in section 6, (6.15) that the creation (annihilation) operators commute among themselves:

$$
\left[B_{k}^{+}, B_{l}^{+}\right]=\left[B_{k}^{-}, B_{l}^{-}\right]=0, \quad k, l \in\left\{f_{j} \mid j=1, \ldots, r\right\}
$$

so that the state (5.1) does not depend on the order of the creation.

The proof is very simple. By using (4.15) we obtain

$$
\frac{d}{d t}\left(L^{ \pm}\right)^{n}=i\left[\mathcal{H},\left(L^{ \pm}\right)^{n}\right]=\left[\left(L^{ \pm}\right)^{n}, M\right] \pm i n \omega\left(L^{ \pm}\right)^{n},
$$

\footnotetext{
${ }^{3}$ We adopt the notation by Olshanetsky and Perelomov [5, 12].
} 
from which

$$
\left[\mathcal{H}, B_{n}^{ \pm}\right]= \pm n \omega B_{n}^{ \pm},
$$

follows after taking the total sum. This simply says that $B_{n}^{ \pm}$creates (annihilates) a state having energy $n w$. In other words we have

$$
\hat{\mathcal{H}} \prod_{j=1}^{r}\left(B_{f_{j}}^{+}\right)^{n_{j}} e^{W}=\left(\mathcal{E}_{0}+\omega \sum_{k=1}^{r} n_{k} f_{k}\right) \prod_{j=1}^{r}\left(B_{f_{j}}^{+}\right)^{n_{j}} e^{W} .
$$

Moreover, it is trivial to show that

$$
\sum_{\nu \in \mathcal{R}}\left(L^{-}\right)_{\mu \nu} e^{W}=\left(p \cdot \mu-i \omega q \cdot \mu+i \sum_{\rho \in \Delta_{+}} \frac{\rho \cdot \mu}{\rho \cdot q}\right) e^{W}=\mu \cdot\left(p+i \frac{\partial W}{\partial q}\right) e^{W}=0,
$$

which implies that the ground state is annihilated by all the annihilation operators

$$
B_{f_{j}}^{-} e^{W}=0, \quad j=1, \ldots, r
$$

Some remarks are in order.

1. In most cases the energy levels are highly degenerate. The above basis is neither orthogonal nor normalised.

2. The independence of the creation-annihilation operators can also be shown in a similar way to that of the conserved quantities. As with the conserved quantities, plural representations are necessary to define the full set of creation-annihilation operators in some models. This aspect will be discussed in later sections in connection with the $\ell$ operators.

3. Reflecting the universality of the first exponent, $f_{1}=2$, the creation and annihilation operators of the least quanta, $2 \omega$, exist in all the models. They form an $\operatorname{sl}(2, \mathbf{R})$ algebra together with the Hamiltonian $\hat{\mathcal{H}}$ :

$$
\left[\hat{\mathcal{H}}, b_{2}^{ \pm}\right]= \pm 2 \omega b_{2}^{ \pm}, \quad\left[b_{2}^{+}, b_{2}^{-}\right]=-\omega^{-1} \hat{\mathcal{H}}
$$

in which $b_{2}^{ \pm}$are normalised forms of $B_{2}^{ \pm}$:

$$
b_{2}^{ \pm}=\sum_{\mu, \nu \in \mathcal{R}}\left(L^{ \pm}\right)_{\mu \nu}^{2} /\left(4 \omega C_{\mathcal{R}}\right) .
$$

The $\operatorname{sl}(2, \mathbf{R})$ algebra was discussed by many authors (see, for example, [12, 30, 17, 21] and others) in connection with the models based on classical root systems. We will 
show later in subsection 7.2 that the states created by $B_{2}^{+}\left(b_{2}^{+}\right)$only can be expressed by the Laguerre polynomial:

$$
\left(b_{2}^{+}\right)^{n} e^{W}=n ! L_{n}^{\left(\tilde{\mathcal{E}}_{0}-1\right)}\left(\omega q^{2}\right) e^{W}, \quad \tilde{\mathcal{E}}_{0} \equiv \mathcal{E}_{0} / \omega
$$

It is trivial to verify that $L_{n}^{\left(\tilde{\mathcal{E}}_{0}-1\right)}\left(\omega q^{2}\right)$ is an eigenfunction of $\tilde{\mathcal{H}}(3.5)$

$$
\tilde{\mathcal{H}} L_{n}^{\left(\tilde{\mathcal{E}}_{0}-1\right)}\left(\omega q^{2}\right)=2 n \omega L_{n}^{\left(\tilde{\mathcal{E}}_{0}-1\right)}\left(\omega q^{2}\right)
$$

The normalisation of the state

$$
\left\|\left(b_{2}^{+}\right)^{n} e^{W}\right\|^{2}=n ! \mathcal{N}_{0} / \Gamma\left(n+\tilde{\mathcal{E}}_{0}\right), \quad \mathcal{N}_{0} \equiv\left\|e^{W}\right\|^{2} \Gamma\left(\tilde{\mathcal{E}}_{0}\right),
$$

is also dictated by the $\operatorname{sl}(2, \mathbf{R})$ relations. The Laguerre polynomial wavefunctions appear as 'radial' wavefunctions in all the cases [31]. This will be shown explicitly for for the rank two models given in subsection 7.3 .

4. As is emphasised by Perelomov [12] and Gambardella [30 the $\operatorname{sl}(2, \mathbf{R})$ algebra and the corresponding Laguerre wavefunctions are more universal than Calogero-Moser models. They arise when the potentials are homogeneous functions in $q$ of degree -2 with the confining harmonic force.

5. The operators $\left\{Q_{n}\right\}$ and $\left\{B_{n}^{ \pm}\right\}$do not form a Lie algebra. They satisfy interesting non-linear relations, for example,

$$
\left[\left[B_{n}^{+}, b_{2}^{-}\right], b_{2}^{+}\right]=n B_{n}^{+}, \quad\left[\left[B_{n}^{-}, b_{2}^{+}\right], b_{2}^{-}\right]=n B_{n}^{-} .
$$

This tells, for example, that although $B_{n}^{+}$and $b_{2}^{+}$create different units of quanta $n$ and 2 , they are not independent

$$
\left[B_{n}^{+}, b_{2}^{-}\right] \neq 0 \neq\left[B_{n}^{-}, b_{2}^{+}\right]
$$

Clarification of the algebraic structure (5.15) for each root system is wanted.

\section{$6 \ell$ operators}

In this section we will show the equivalence of the quantum conserved quantities obtained in the Lax operator formalism of section 4 and those derived in the 'commuting differential 
operators' formalism initiated by Dunkl [10] and followed by many authors. Again the equivalence is universal, applicable to the models based on any root systems. We propose to call the operators in the latter approach simply ' $\ell$ operators', since they are essentially the same as the $L$ operator in the Lax pair formalism and that they are not mutually commuting, as we will show presently, when the interaction potentials are trigonometric (hyperbolic), (6.14). Although these two formalisms are formally equivalent, the $\ell$ operator formalism has many advantages over the Lax pair one. Roughly speaking, the 'vector-like' objects $\ell_{\mu}$ 's are easier to handle than the matrix $L_{\mu \nu}$.

Let us fix a representation $\mathcal{R}$ of the Coxeter group $G_{\Delta}$ and define for each element $\mu \in \mathcal{R}$ the following differential-reflection operator

$$
\ell_{\mu}=\ell \cdot \mu=p \cdot \mu+i \sum_{\rho \in \Delta_{+}} g_{|\rho|}(\rho \cdot \mu) x(\rho \cdot q) \check{s}_{\rho}, \quad \mu \in \mathcal{R}
$$

It is linear in $\mu$ and Coxeter covariant

$$
\ell_{\mu+\nu}=\ell_{\mu}+\ell_{\nu}, \quad \check{s}_{\rho} \ell_{\mu} \check{s}_{\rho}=\ell_{s_{\rho}(\mu)}, \quad \forall \rho \in \Delta .
$$

They are hermitian operators, $\ell_{\mu}^{\dagger}=\ell_{\mu}$, with respect to the standard inner product for the states (5.4).

It is straightforward to show that the quantum conserved quantities $Q_{n}$ derived in the previous section (4.7) can be expressed as polynomials in the $\ell$ operators as follows:

$$
Q_{n} \psi=\sum_{\mu, \nu \in \mathcal{R}}\left(L^{n}\right)_{\mu \nu} \psi=\left(\sum_{\mu \in \mathcal{R}} \ell_{\mu}^{n}\right) \psi
$$

in which $\psi$ is an arbitrary Coxeter invariant state, $\check{s}_{\rho} \psi=\psi$. This also illustrates the Coxeter invariance of $Q_{n}$ clearly, since $\check{s}_{\rho}\left(\sum_{\mu \in \mathcal{R}} \ell_{\mu}^{n}\right) \check{s}_{\rho}=\sum_{\mu \in \mathcal{R}} \ell_{s_{\rho}(\mu)}^{n}=\sum_{\mu \in \mathcal{R}} \ell_{\mu}^{n}$. For $n=1$ it is trivial, since

$$
\begin{aligned}
\sum_{\nu \in \mathcal{R}}(L)_{\mu \nu} \psi & =\left(p \cdot \mu+i \sum_{\rho \in \Delta_{+}} g_{|\rho|}(\rho \cdot \mu) x(\rho \cdot q) \sum_{\nu \in \mathcal{R}}\left(\hat{s}_{\rho}\right)_{\mu \nu}\right) \psi \\
& =\left(p \cdot \mu+i \sum_{\rho \in \Delta_{+}} g_{|\rho|}(\rho \cdot \mu) x(\rho \cdot q) \check{s}_{\rho}\right) \psi=\ell_{\mu} \psi,
\end{aligned}
$$

in which $\sum_{\nu \in \mathcal{R}}\left(\hat{s}_{\rho}\right)_{\mu \nu}=1$ and $\check{s}_{\rho} \psi=\psi$ are used. Let us assume that

$$
\sum_{\nu \in \mathcal{R}}\left(L^{n}\right)_{\mu \nu} \psi=\ell_{\mu}^{n} \psi
$$


is correct, then we obtain

$$
\begin{gathered}
\sum_{\nu \in \mathcal{R}}\left(L^{n+1}\right)_{\mu \nu} \psi=\sum_{\lambda, \nu \in \mathcal{R}} L_{\mu \lambda}\left(L^{n}\right)_{\lambda \nu} \psi=\sum_{\lambda \in \mathcal{R}} L_{\mu \lambda} \ell_{\lambda}^{n} \psi, \\
=\sum_{\lambda \in \mathcal{R}}\left(p \cdot \mu \delta_{\mu \lambda}+i \sum_{\rho \in \Delta_{+}} g_{|\rho|}(\rho \cdot \mu) x(\rho \cdot q)\left(\hat{s}_{\rho}\right)_{\mu \lambda}\right) \ell_{\lambda}^{n} \psi .
\end{gathered}
$$

In the second summation only such $\lambda$ as $\lambda=s_{\rho}(\mu)$ contributes and we find

$$
\ell_{s_{\rho}(\mu)}^{n} \psi=\left(\check{s}_{\rho} \ell_{\mu}^{n} \check{s}_{\rho}\right) \psi=\check{s}_{\rho} \ell_{\mu}^{n} \psi .
$$

Thus we arrive at

$$
\sum_{\nu \in \mathcal{R}}\left(L^{n+1}\right)_{\mu \nu} \psi=\ell_{\mu}^{n+1} \psi
$$

and the equivalence of the two expressions of the conserved quantity (6.3) is proved.

Commutation relations among $\ell$ operators can be evaluated in a similar manner as those appearing in the Lax pair [7. 9], that is, by decomposing the roots into two-dimensional sub-root systems. We obtain

$$
\left[\ell_{\mu}, \ell_{\nu}\right]=-a^{2} \sum_{\rho, \sigma \in \Delta_{+}} g_{|\rho|} g_{|\sigma|}(\rho \cdot \mu)(\sigma \cdot \nu)\left[\check{s}_{\rho}, \check{s}_{\sigma}\right] \times\left\{\begin{aligned}
0 & \text { rational, } \\
-1 & \text { hyperbolic, } \\
1 & \text { trigonometric. }
\end{aligned}\right.
$$

One important use of the $\ell$ operators is the proof of involution of quantum conserved quantities. For type I models Heckman [21] gave a universal proof based on the commutation relation (6.7):

$$
\left[Q_{n}, Q_{m}\right] \psi=\sum_{\mu, \nu \in \mathcal{R}}\left[\ell_{\mu}^{n}, \ell_{\nu}^{m}\right] \psi=0, \quad \text { rational model. }
$$

This was the motivation for the introduction of the commuting differential-reflection operators by Dunkl, [10]. In fact, Dunkl's and Heckman's operators were the similarity transformation of $\ell_{\mu}$ by the ground state wavefunction $e^{W}$ :

$$
\tilde{\ell}_{\mu}=e^{-W} \ell_{\mu} e^{W}=p \cdot \mu+i \sum_{\rho \in \Delta_{+}} g_{|\rho|} \frac{(\rho \cdot \mu)}{(\rho \cdot q)}\left(\check{s}_{\rho}-1\right) .
$$

As for type $\mathrm{V}$ models, we define $\ell^{ \pm}$corresponding to $L^{ \pm}$(4.16):

$$
\ell_{\mu}^{ \pm}=\ell^{ \pm} \cdot \mu=p \cdot \mu \pm i \omega(q \cdot \mu)+i \sum_{\rho \in \Delta_{+}} g_{|\rho|} \frac{(\rho \cdot \mu)}{(\rho \cdot q)} \check{s}_{\rho}, \quad \mu \in \mathcal{R} .
$$

They are linear in $\mu$, Coxeter covariant and hermitian conjugate of each other with respect to the standard inner product (5.4):

$$
\check{s}_{\rho} \ell_{\mu}^{ \pm} \check{s}_{\rho}=\ell_{s_{\rho}(\mu)}^{ \pm}, \quad\left(\ell_{\mu}^{ \pm}\right)^{\dagger}=\ell_{\mu}^{\mp} .
$$


The conserved quantities are expressed as polynomials in $\ell^{ \pm}$operators:

$$
\begin{aligned}
\operatorname{Ts}\left(\mathcal{L}_{1}^{n}\right) \psi & =\sum_{\mu, \nu \in \mathcal{R}}\left(L^{+} L^{-}\right)_{\mu \nu}^{n} \psi=\sum_{\mu \in \mathcal{R}}\left(\ell_{\mu}^{+} \ell_{\mu}^{-}\right)^{n} \psi \\
\operatorname{Ts}\left(\mathcal{L}_{2}^{n}\right) \psi & =\sum_{\mu, \nu \in \mathcal{R}}\left(L^{-} L^{+}\right)_{\mu \nu}^{n} \psi=\sum_{\mu \in \mathcal{R}}\left(\ell_{\mu}^{-} \ell_{\mu}^{+}\right)^{n} \psi
\end{aligned}
$$

Likewise the creation and annihilation operators $B_{n}^{ \pm}(5.2)$ are expressed as

$$
B_{n}^{ \pm} \psi=\operatorname{Ts}\left(L^{ \pm}\right)^{n} \psi=\sum_{\mu, \nu \in \mathcal{R}}\left(L^{ \pm}\right)_{\mu \nu} \psi=\sum_{\mu \in \mathcal{R}}\left(\ell_{\mu}^{ \pm}\right)^{n} \psi
$$

The commutation relations among $\ell^{ \pm}$operators are easy to evaluate, since $\ell$ operators commute in the rational potential models (6.7):

$$
\left[\ell_{\mu}^{+}, \ell_{\nu}^{+}\right]=\left[\ell_{\mu}^{-}, \ell_{\nu}^{-}\right]=0, \quad\left[\ell_{\mu}^{-}, \ell_{\nu}^{+}\right]=2 \omega\left(\mu \cdot \nu+\sum_{\rho \in \Delta_{+}} g_{|\rho|}(\rho \cdot \mu)\left(\rho^{\vee} \cdot \nu\right) \check{s}_{\rho}\right)
$$

From these it follows that the creation (annihilation) operators $B_{n}^{ \pm}$do commute among themselves:

$$
\left[B_{n}^{+}, B_{m}^{+}\right] \psi=\left[B_{n}^{-}, B_{m}^{-}\right] \psi=0
$$

It is also clear that $\ell_{\mu}^{ \pm} / \sqrt{2 \omega}$ are the 'deformation' of the creation (annihilation) operators of the ordinary multicomponent harmonic oscillators. In fact we have

$$
\ell_{\mu}^{+} e^{W}=2 i \omega(\mu \cdot q) e^{W} \text { and } \ell_{\mu}^{-} e^{W}=0
$$

In the next section we present an alternative scheme of algebraic construction of excited states of type $\mathrm{V}$ models by pursuing the analogy that $\ell^{ \pm}$are the creation and annihilation operators of the unit quantum. This method was applied to the $A_{r}$ models by Brink et. al and others [17, 15, 18].

\section{Algebraic construction of excited states II}

\subsection{Operator solution of the triangular Hamiltonian}

In subsection 3.1, we have shown that an eigenfunction of $\mathcal{H}$ with eigenvalue $N \omega$ is given by

$$
\left(P_{N}(q)+\tilde{P}_{N-2}(q)\right) e^{W}
$$

in which $P_{N}(q)$ is a Coxeter invariant polynomial in $q$ of homogeneous degree $N$ and $\tilde{P}_{N-2}(q)$ is a Coxeter invariant polynomial in $q$ of degree $N-2$ and lower. The non-leading polynomial 
$\tilde{P}_{N-2}(q)$ is completely determined by the leading one $P_{N}(q)$ due to the triangularity. This solution can be written in an operator form as follows.

Suppose $P_{N}(q)$ is expressed as

$$
P_{N}(q)=\sum_{\{\mu\}} c_{\{\mu\}}\left(q \cdot \mu_{1}\right) \cdots\left(q \cdot \mu_{N}\right), \quad \mu_{j} \in \mathcal{R}, \quad c_{\{\mu\}}: \text { const. }
$$

We obtain a Coxeter invariant polynomial in the creation operators $\ell^{+}$by replacing $q \cdot \mu$ by $\ell_{\mu}^{+} /(2 i \omega)$ :

$$
P_{N}(q) \Rightarrow \frac{1}{(2 i \omega)^{N}} P_{N}\left(\ell^{+}\right)
$$

This creates the above eigenfunction of $\mathcal{H}$ from the ground state:

$$
\frac{1}{(2 i \omega)^{N}} P_{N}\left(\ell^{+}\right) e^{W}=\left(P_{N}(q)+\tilde{P}_{N-2}(q)\right) e^{W} .
$$

The proof is again elementary. By using the commutation relations among $\ell^{ \pm}$operators it is straightforward to derive the explicit expression of the Hamiltonian in terms of $\ell^{ \pm}$:

$$
\mathcal{H}=\frac{1}{2 \mathcal{C}_{\mathcal{R}}} \sum_{\mu \in \mathcal{R}} \ell_{\mu}^{+} \ell_{\mu}^{-}+\sum_{\rho \in \Delta_{+}} g_{|\rho|}\left(\omega+\frac{1}{2} \frac{|\rho|^{2}}{(\rho \cdot q)^{2}}\right)\left(\check{s}_{\rho}-1\right),
$$

in which the second term vanishes upon acting on a Coxeter invariant state. Next we obtain

$$
\frac{1}{2 \mathcal{C}_{\mathcal{R}}} \sum_{\mu \in \mathcal{R}}\left[\ell_{\mu}^{+} \ell_{\mu}^{-}, \ell_{\nu}^{ \pm}\right]=\left[\ell_{\nu}^{ \pm}, S\right] \pm \omega \ell_{\nu}^{ \pm}, \quad S \equiv \sum_{\rho \in \Delta_{+}} g_{|\rho|} \check{s}_{\rho},
$$

which is an $\ell$ operator version of (4.15). Since a commutator is a derivation, we obtain

$$
\frac{1}{2 \mathcal{C}_{\mathcal{R}}} \sum_{\mu \in \mathcal{R}}\left[\ell_{\mu}^{+} \ell_{\mu}^{-}, P_{N}\left(\ell^{+}\right)\right]=\left[P_{N}\left(\ell^{+}\right), S\right]+N \omega P_{N}\left(\ell^{+}\right),
$$

in which the first term in r.h.s. vanishes due to the Coxeter invariance of $P_{N}$. Thus we arrive at the desired commutation relation

$$
\left[\mathcal{H}, P_{N}\left(\ell^{+}\right)\right]=N \omega P_{N}\left(\ell^{+}\right)+\sum_{\rho \in \Delta_{+}} g_{|\rho|}\left[\frac{1}{2} \frac{|\rho|^{2}}{(\rho \cdot q)^{2}}, P_{N}\left(\ell^{+}\right)\right]\left(\check{s}_{\rho}-1\right),
$$

and the eigenvalue equation

$$
\mathcal{H} P_{N}\left(\ell^{+}\right) e^{W}=N \omega P_{N}\left(\ell^{+}\right) e^{W}
$$

Since the action of the creation operators on the ground state is

$$
\ell_{\mu_{1}}^{+} \cdots \ell_{\mu_{N}}^{+} e^{W}=\left[(2 i \omega)^{N}\left(q \cdot \mu_{1}\right) \cdots\left(q \cdot \mu_{N}\right)+\text { lower powers of } q\right] e^{W}
$$


our assertion (7.3) is proved. It should be stressed that in this formalism the Coxeter invariance of the polynomial $P$ is important but not how it is obtained.

Like the above Hamiltonian (7.4), the $\ell$ operator formulas of higher conserved quantities (6.12) contain extra terms:

$$
\operatorname{Ts}\left(\mathcal{L}_{1}^{n}\right)=\sum_{\mu, \nu \in \mathcal{R}}\left(L^{+} L^{-}\right)_{\mu \nu}^{n}=\sum_{\mu \in \mathcal{R}}\left(\ell_{\mu}^{+} \ell_{\mu}^{-}\right)^{n}+V T
$$

Here VT stands for vanishing terms when they act on a Coxeter invariant state. The same is true for most formulas derived in section 6 .

\subsection{States Created by $B_{2}^{+}$}

Here we derive the explicit forms of the subseries of eigenstates obtained by multiple applications of the least quanta creation operator $B_{2}^{+}$(5.2), or its normalised form $b_{2}^{+}$(5.11). It is convenient to work with the similarity transformed operator

$$
\tilde{b}_{2}^{+}=e^{-W} b_{2}^{+} e^{W}=\frac{1}{4 \omega C_{\mathcal{R}}} \sum_{\mu \in \mathcal{R}}\left(\tilde{\ell}_{\mu}^{+}\right)^{2}+V T,
$$

in which

$$
\tilde{\ell}_{\mu}^{+}=p \cdot \mu+2 i \omega(q \cdot \mu)+i \sum_{\rho \in \Delta_{+}} \frac{\rho \cdot \mu}{\rho \cdot q}\left(\check{s}_{\rho}-1\right) .
$$

Let $f(u)$ be an arbitrary function of $u \equiv \omega q^{2}$, then it is Coxeter invariant. We find

$$
\tilde{\ell}_{\mu}^{+} f(u)=2 i \omega(q \cdot \mu)\left(1-\frac{d}{d u}\right) f(u), \quad u \equiv \omega q^{2},
$$

and

$$
\tilde{b}_{2}^{+} f(u)=-\left[u\left(1-\frac{d}{d u}\right)^{2}-\tilde{\mathcal{E}}_{0}\left(1-\frac{d}{d u}\right)\right] f(u) .
$$

Since $\tilde{b}_{2}^{+} 1=\tilde{\mathcal{E}}_{0}-u=L_{1}^{\left(\tilde{\mathcal{E}}_{0}-1\right)}(u)$, we assume

$$
\left(\tilde{b}_{2}^{+}\right)^{n} 1=n ! L_{n}^{\left(\tilde{\mathcal{E}}_{0}-1\right)}(u)
$$

By using the Laguerre differential equation (3.21) and the recurrence formulas of the Laguerre polynomial $L_{n}^{(\alpha)}(u)$,

$$
\begin{aligned}
& u \frac{d}{d u} L_{n}^{(\alpha)}(u)=n L_{n}^{(\alpha)}(u)-(n+\alpha) L_{n-1}^{(\alpha)}(u) \\
& n L_{n}^{(\alpha)}(u)+(u-2 n-\alpha+1) L_{n-1}^{(\alpha)}(u)+(n+\alpha-1) L_{n-2}^{(\alpha)}(u)=0
\end{aligned}
$$


we can show

$$
-\left[u\left(1-\frac{d}{d u}\right)^{2}-\tilde{\mathcal{E}}_{0}\left(1-\frac{d}{d u}\right)\right] L_{n}^{\left(\tilde{\mathcal{E}}_{0}-1\right)}(u)=(n+1) L_{n+1}^{\left(\tilde{\mathcal{E}}_{0}-1\right)}(u) .
$$

Thus the induction is proved and we arrive at (5.12). The orthogonality of the states

$$
\left(\left(B_{2}^{+}\right)^{n} e^{W},\left(B_{2}^{+}\right)^{m} e^{W}\right)=0, \quad n \neq m
$$

can be easily understood as the $d u$ part of the measure

$$
e^{2 W} d^{r} q=e^{-u} u^{\tilde{\mathcal{E}}_{0}-1} d u d \Omega, \quad d \Omega: \text { angular part }
$$

is the proper weight function for the Laguerre polynomial $L_{n}^{\left(\tilde{\mathcal{E}}_{0}-1\right)}(u)$.

\subsection{Explicit solutions of the rank two models}

For rank two models, the Liouville integrability, or the involution of conserved quantities is automatically satisfied since the second conserved quantity is already obtained. For rank two type $\mathrm{V}$ models, the complete set of orthogonal wavefunctions can be written down explicitly in terms of separation of variables by using the Coxeter invariant polynomials. These are based on the dihedral root systems $I_{2}(m)$, with $A_{2} \cong I_{2}(3)$ [31], $B_{2} \cong I_{2}(4)$ and $G_{2} \cong I_{2}(6)$ [32. The Coxeter invariant polynomials exist at degree 2, i.e. $q^{2}$ and $m$ which is

$$
\prod_{j=1}^{m}\left(v_{j} \cdot q\right)
$$

where $\left\{v_{j}\right\}$ is a set of vectors given in $(\underline{B .3})$. If we introduce the two-dimensional polar coordinates system for $q$

$$
q=r(\sin \theta, \cos \theta)
$$

then the principal Weyl chamber is

$$
P W: 0<r^{2}<\infty, \quad 0<\theta<\pi / m
$$

The two Coxeter invariant variables read:

$$
q^{2}=r^{2}, \quad \prod_{j=1}^{m}\left(v_{j} \cdot q\right)=2\left(\frac{r}{2}\right)^{m} \cos m \theta
$$

\footnotetext{
${ }^{4}$ We believe no confusion arises here, between the radial coordinate variable $r$ and the rank of the root system $r$, which in this case is 2 of $I_{2}(m)$.
} 
and the latter variable varies the full range, $-1<\cos m \theta<1$ in the $P W$. Thus solving the eigenvalue equation for $\tilde{\mathcal{H}}$ (3.6) by separation of variables in the polar coordinate system is compatible with Coxeter invariance. We adopt as two independent variables

$$
u \equiv \omega r^{2}, \quad z \equiv \cos m \theta
$$

The solutions consist of a Gegenbauer (Jacobi) polynomial in $\cos m \theta$ times a Laguerre polynomial in $\omega r^{2}$. The former we have encountered in the $A_{1}$ Sutherland problem, subsection 3.2 and the latter in the $A_{1}$ Calogero problem subsections 3.1 and 7.2 .

Let us demonstrate this for odd $m$ with a single coupling constant and for even $m$ with two independent coupling constants, in parallel. In terms of the Coxeter invariant variables (7.24) the $I_{2}(m)$ Hamiltonians take surprisingly simple forms:

$$
\begin{aligned}
\tilde{\mathcal{H}} & =\omega r \frac{\partial}{\partial r}-\frac{1}{2 r} \frac{\partial}{\partial r}\left(r \frac{\partial}{\partial r}\right)-\frac{1}{2 r^{2}}\left[\frac{\partial^{2}}{\partial \theta^{2}}+m\left\{\begin{array}{c}
2 g \cot m \theta \\
\left.\left.-g_{0} \tan \frac{m \theta}{2}+g_{e} \cot \frac{m \theta}{2}\right\} \frac{\partial}{\partial \theta}\right] \\
\end{array}=-2 \omega\left[u \frac{\partial^{2}}{\partial u^{2}}+(1-u) \frac{\partial}{\partial u}\right]-\frac{\omega m^{2}}{2 u}\left[\left(1-z^{2}\right) \frac{\partial^{2}}{\partial z^{2}}+\left\{\begin{array}{c}
0 \\
g_{o}-g_{e}
\end{array}\right\}-\left\{\begin{array}{c}
1+2 g \\
1+g_{e}+g_{o}
\end{array}\right\} z \frac{\partial}{\partial z}\right] .\right.\right.
\end{aligned}
$$

The $z$ part admits polynomial solutions

$$
\begin{aligned}
{\left[\left(1-z^{2}\right) \frac{d^{2}}{d z^{2}}+\left\{\begin{array}{c}
0 \\
g_{o}-g_{e}
\end{array}\right\}\right.} & \left.-\left\{\begin{array}{c}
1+2 g \\
1+g_{e}+g_{o}
\end{array}\right\} z \frac{d}{d z}\right] P_{\ell}^{\left\{\begin{array}{c}
\left(g-\frac{1}{2}, g-\frac{1}{2}\right) \\
\left(g_{o}-\frac{1}{2}, g_{e}-\frac{1}{2}\right)
\end{array}\right\}}(z) \\
& =-\ell\left(\ell+\left\{\begin{array}{c}
2 g \\
g_{e}+g_{o}
\end{array}\right\}\right) P_{\ell}^{\left\{\begin{array}{c}
\left(g-\frac{1}{2}, g-\frac{1}{2}\right) \\
\left(g_{o}-\frac{1}{2}, g_{e}-\frac{1}{2}\right)
\end{array}\right\}}(z),
\end{aligned}
$$

in which $\ell$ is the degree of the polynomial. After substituting them, the radial part of the Hamiltonian $\tilde{\mathcal{H}}_{r}$ reads

$$
\tilde{\mathcal{H}}_{r}=-2 \omega\left[u \frac{d^{2}}{d u^{2}}+(1-u) \frac{d}{d u}-\frac{m^{2}}{4 u} \ell\left(\ell+\left\{\begin{array}{c}
2 g \\
g_{e}+g_{o}
\end{array}\right\}\right)\right] .
$$

By similarity transformation in terms of $u^{m \ell / 2} \propto r^{m \ell}$, which is the radial part of the highest term of the polynomial $P_{\ell}^{(\alpha, \beta)}\left(r^{m} \cos m \theta\right)$, it reads

$$
u^{-m \ell / 2} \tilde{\mathcal{H}}_{r} u^{m \ell / 2}=-2 \omega\left[u \frac{d^{2}}{d u^{2}}+\left(m\left(\left\{\begin{array}{c}
\ell+g \\
\ell+\frac{1}{2}\left(g_{e}+g_{o}\right)
\end{array}\right\}\right)+1-u\right) \frac{d}{d u}-\frac{m \ell}{2}\right] .
$$

This is the main part of the differential equation for the Laguerre polynomial (3.21):

$$
\left[u \frac{d^{2}}{d u^{2}}+\left(m \ell+\tilde{\mathcal{E}}_{0}-u\right) \frac{d}{d u}-n\right] L_{n}^{\left(m \ell+\tilde{\mathcal{E}}_{0}-1\right)}(u)=0
$$


in which the indices $m(\ell+g)$ and $m\left(\ell+\left(g_{o}+g_{e}\right) / 2\right)$ can be written in a unified way as $m \ell+\tilde{\mathcal{E}}_{0}-1$. Thus the eigenstates of the Hamiltonian are obtained:

$$
\begin{aligned}
& \tilde{\mathcal{H}}_{r} u^{m \ell / 2} L_{n}^{\left(m \ell+\tilde{\mathcal{E}}_{0}-1\right)}(u)=\omega(2 n+m \ell) u^{m \ell / 2} L_{n}^{\left(m \ell+\tilde{\mathcal{E}}_{0}-1\right)}(u), \\
& \hat{\mathcal{H}} u^{m \ell / 2} L_{n}^{\left(m \ell+\tilde{\mathcal{E}}_{0}-1\right)}(u) P_{\ell}^{\left\{\begin{array}{c}
\left(g-\frac{1}{2}, g-\frac{1}{2}\right) \\
\left(g_{o}-\frac{1}{2}, g_{e}-\frac{1}{2}\right)
\end{array}\right\}}(z) \\
& =\left(\omega(2 n+m \ell)+\mathcal{E}_{o}\right) u^{m \ell / 2} L_{n}^{\left(m \ell+\tilde{\mathcal{E}}_{0}-1\right)}(u) P_{\ell}^{\left\{\begin{array}{c}
\left(g-\frac{1}{2}, g-\frac{1}{2}\right) \\
\left(g_{o}-\frac{1}{2}, g_{e}-\frac{1}{2}\right)
\end{array}\right\}}(z) .
\end{aligned}
$$

It is instructive to note that the Hamiltonians $\hat{\mathcal{H}}$ look also simple:

$$
\hat{\mathcal{H}}=\omega r \frac{\partial}{\partial r}-\frac{1}{2 r} \frac{\partial}{\partial r}\left(r \frac{\partial}{\partial r}\right)-\frac{1}{2 r^{2}} \frac{\partial^{2}}{\partial \theta^{2}}-\frac{m^{2}}{2 r^{2}}\left\{\begin{array}{c}
\frac{g(g-1)}{\sin ^{2} m \theta} \\
\frac{g_{o}\left(g_{o}-1\right)}{4 \cos ^{2} \frac{m \theta}{2}}+\frac{g_{e}\left(g_{e}-1\right)}{4 \sin ^{2} \frac{m \theta}{2}}
\end{array}\right\}
$$

Olshanetsky and Perelomov [5] obtained the above solutions starting from these formulas.

\section{Involution of conserved quantities}

\subsection{Universal proof of involution of quantum conserved quantities for type I, II and III models}

Here we present a proof of involution of quantum conserved quantities $\left\{Q_{n}\right\}$ derived from the universal Lax pair in subsection 4.1 for type I, II and III models. The proof is applicable to all models based on any root systems. Though a universal proof of involution for type I models is given by Heckman [21] as recapitulated in section 6, we believe the universal proof applicable to type II and III models as well is new. It depends on a theorem by Olshanetsky and Perelomov [11]. Our own contribution is that we have provided a universal Lax pair and conserved quantities satisfying all the requirements of the theorem.

Liouville's theorem states the complete integrability as the existence of an involutive set of conserved quantities as many as the degrees of freedom. We have already given conserved quantities $\left\{Q_{n}\right\}$ (4.7) independent and as many as the degrees of freedom (see Appendix B). They have the following properties:

1. Coxeter invariance

$$
Q_{n}\left(s_{\rho}(p), s_{\rho}(q)\right)=Q_{n}(p, q), \quad \forall \rho \in \Delta
$$

2. $Q_{n}(p, q)$ is a homogeneous polynomial of degree $n$ in variables $\left(p_{1}, \ldots, p_{r}, x(\rho \cdot q)\right)$. 
3. Scaling property for those of type I models:

$$
{ }^{I} Q_{n}\left(\kappa^{-1} p, \kappa q\right)=\kappa^{-n I} Q_{n}(p, q)
$$

as a consequence of the above point.

4. For type II and III models, the asymptotic behaviour near the origin:

$$
Q_{n}(p, q)={ }^{I} Q_{n}(p, q)(1+\mathcal{O}(|q|)), \quad \text { for } \quad|q| \rightarrow 0 .
$$

We need to show the vanishing of

$$
J_{l m} \equiv\left[Q_{l}, Q_{m}\right]
$$

which is a polynomial in $\{p\}$ of degree $s$

$$
s<l+m \text {. }
$$

Let us decompose $J_{l m}$ into the leading part and the rest:

$$
J_{l m}=J_{l m}^{0}+J_{l m}^{r e s t}, \quad J_{l m}^{0}=\sum c^{j_{1}, \ldots, j_{s}}(q) p_{j_{1}} \ldots p_{j_{s}}
$$

and $J_{l m}^{\text {rest }}$ is a polynomial in $\{p\}$ of degree less than $s$. From Jacobi identity and conservation $\left[\mathcal{H}, Q_{l(m)}\right]=0$, we obtain

$$
\left[\mathcal{H}, J_{l m}\right]=0 .
$$

Considering the explicit form of the Hamiltonian (2.7) $(\omega=0)$, the leading (i.e. of degree $s+1$ in $\{p\})$ part of $\left[\mathcal{H}, J_{l m}\right]$ comes only from the free part

$$
\left[p^{2}, J_{l m}^{0}\right]
$$

and it vanishes if the following conditions are satisfied:

$$
\sum_{\sigma} \frac{\partial}{\partial q_{t}} c^{k_{1}, \ldots, k_{s}}(q)=0
$$

where the sum is taken over all permutations of indices $\sigma\left(t, k_{1}, \ldots, k_{s}\right)=\left(j_{1}, \ldots, j_{s+1}\right)$. In [33 it is proved (Lemma 2.5, p. 407) that the system (8.8) has only polynomial solutions. Then Olshanetsky and Perelomov argue that for type I models the scaling property tells that $c^{k_{1}, \ldots, k_{s}}(\kappa q)=\kappa^{s-l-m} c^{k_{1}, \ldots, k_{s}}(q)$. Since $s<l+m$ (8.5), it follows that the only polynomial solution satisfying the condition is the null polynomial. Thus we obtain $c^{j_{1}, \ldots, j_{s}}(q)=0$ $\Rightarrow J_{l m}^{0}=0$ and $J_{l m}=0$. The same results follow for type II and III models by considering the asymptotic behaviour for $|q| \rightarrow 0$. Thus the involution of all the conserved quantities $\left\{Q_{n}\right\}$ is proved. This result also implies the involution of classical conserved quantities by taking the classical limit $(\hbar \rightarrow 0)$. 


\subsection{Rational models with the harmonic confining force}

In this subsection we show the involution of quantum conserved quantities for the type $\mathrm{V}$ models based on the root systems of classical Lie algebras. The method is a straightforward generalisation of the one developed by Polychronakos on the $A_{r}$ model. This is made possible by the availability of the universal Lax pair formalism [7, 9], in particular the root type and minimal type Lax pairs. We apply it to the models based on $B_{r}$ and $D_{r}$ root systems. For the rational potential, the $B_{r}, C_{r}$ and $B C_{r}$ models are equivalent. Let us choose $\mathcal{R}=\left\{ \pm e_{j} \in\right.$ $\left.\mathbf{R}^{r} \mid e_{j} \cdot e_{k}=\delta_{j k}\right\}$ as the representation space of the Coxeter group consisting of orthogonal vectors and their negatives. They are the set of short roots of $B_{r}$ and the set of vector weights of $D_{r}$ in the parametrisation of roots given in (B.1) and (4.13), respectively.

The conserved quantities in the $\ell$ operator form are given by

$$
Q_{n}={ }^{\ell} Q_{n}+V T, \quad{ }^{\ell} Q_{n} \equiv \sum_{j=1}^{r}\left(\ell_{j}^{+} \ell_{j}^{-}\right)^{n}, \quad n=1, \ldots, r,
$$

in which we abbreviate $\ell_{e_{j}}^{ \pm}$as $\ell_{j}^{ \pm}$. In this case the commutation relation among $\ell$ operators (6.14) are greatly simplified thanks to the orthogonality of $\left\{e_{j}\right\}$ 's and the explicit forms of the roots:

$$
\left[\ell_{j}^{-}, \ell_{k}^{+}\right]=-2 \omega \tilde{g}\left(\check{s}_{j k}-\overline{\check{s}}_{j k}\right)
$$

in which

$$
\tilde{g}=\left\{\begin{array}{cc}
g_{L} & B_{r} \text { model }, \\
g & D_{r} \text { model },
\end{array} \check{s}_{j k} \equiv \check{s}_{e_{j}-e_{k}}, \quad \check{s}_{j k} \equiv \check{s}_{e_{j}+e_{k}} .\right.
$$

By repeating them we obtain

$$
\begin{aligned}
{\left[\ell_{j}^{+} \ell_{j}^{-}, \ell_{k}^{+} \ell_{k}^{-}\right] } & =-2 \omega \tilde{g}\left[\ell_{j}^{+} \ell_{j}^{-}, m_{j k}\right], \quad m_{j k} \equiv \check{s}_{j k}+\bar{s}_{j k}=m_{k j}, \quad j \neq k, \\
{\left[\left(\ell_{j}^{+} \ell_{j}^{-}\right)^{n}, \ell_{k}^{+} \ell_{k}^{-}\right] } & =-2 \omega \tilde{g}\left[\left(\ell_{j}^{+} \ell_{j}^{-}\right)^{n}, m_{j k}\right]=+2 \omega \tilde{g}\left[\left(\ell_{k}^{+} \ell_{k}^{-}\right)^{n}, m_{j k}\right], \\
{\left[\ell_{j}^{+} \ell_{j}^{-},\left(\ell_{k}^{+} \ell_{k}^{-}\right)^{m}\right] } & =+2 \omega \tilde{g}\left[\left(\ell_{k}^{+} \ell_{k}^{-}\right)^{m}, m_{j k}\right]=-2 \omega \tilde{g}\left[\left(\ell_{j}^{+} \ell_{j}^{-}\right)^{m}, m_{j k}\right] .
\end{aligned}
$$

Here and later the identity $\left[\left(\ell_{k}^{+} \ell_{k}^{-}\right)^{t}, m_{j k}\right]=-\left[\left(\ell_{j}^{+} \ell_{j}^{-}\right)^{t}, m_{j k}\right]$ is used repeatedly. Then (8.13) leads to

$$
\left[\left(\ell_{j}^{+} \ell_{j}^{-}\right)^{n},\left(\ell_{k}^{+} \ell_{k}^{-}\right)^{m}\right]=+2 \omega \tilde{g} \sum_{t=0}^{m-1}\left(\left(\ell_{k}^{+} \ell_{k}^{-}\right)^{t+n} m_{j k}\left(\ell_{k}^{+} \ell_{k}^{-}\right)^{m-t-1}-\left(\ell_{k}^{+} \ell_{k}^{-}\right)^{t} m_{j k}\left(\ell_{k}^{+} \ell_{k}^{-}\right)^{m+n-t-1}\right)
$$

and (8.14) to

$$
\left[\left(\ell_{j}^{+} \ell_{j}^{-}\right)^{n},\left(\ell_{k}^{+} \ell_{k}^{-}\right)^{m}\right]=-2 \omega \tilde{g} \sum_{t=0}^{n-1}\left(\left(\ell_{j}^{+} \ell_{j}^{-}\right)^{t+m} m_{j k}\left(\ell_{j}^{+} \ell_{j}^{-}\right)^{n-t-1}-\left(\ell_{j}^{+} \ell_{j}^{-}\right)^{t} m_{j k}\left(\ell_{j}^{+} \ell_{j}^{-}\right)^{m+n-t-1}\right) .
$$


Summing over $j$ and $k$ and adding (8.15) and (8.16) together with the interchange of the dummy indices $j \leftrightarrow k$ in the latter produces

$$
2\left[{ }^{\ell} Q_{n},{ }^{\ell} Q_{m}\right]=+2 \omega \tilde{g} \sum_{t=0}^{n+m-1} \sum_{j, k}\left(\left(\ell_{k}^{+} \ell_{k}^{-}\right)^{t} m_{j k}\left(\ell_{k}^{+} \ell_{k}^{-}\right)^{n+m-t-1}-\left(\ell_{k}^{+} \ell_{k}^{-}\right)^{t} m_{j k}\left(\ell_{k}^{+} \ell_{k}^{-}\right)^{n+m-t-1}\right)=0 .
$$

Thus we obtain

$$
\left[Q_{n}, Q_{m}\right]=0, \quad n, m=1, \ldots, r
$$

on the Fock space of Coxeter invariant states.

\subsection{Lax pair with spectral parameter}

In the theory of classical Calogero-Moser models, Lax pair with spectral parameter $(\xi)$ plays an important role, in particular, in elliptic potential models [34, 8, 6, [7] for derivation of spectral curves, etc. In quantum theory, however, the meaning and use of the spectral parameter are yet to be established, partly because of the underdeveloped stage of the quantum models with elliptic potentials. Here we point out a small use of the quantum Lax pair with spectral parameter in the quantum model with trigonometric potential. Namely, it accounts for the useful trick by Polychronakos [15] for the proof of the involution of conserved quantities in trigonometric $A_{r}$ model. (Now we have a universal proof of involution for type I, II and III models, see the previous subsection.)

From the theory of the generalised Lax pair [7] and its quantum version for degenerate potential models [9], we find that the $L$ operator can contain one additional complex parameter $\xi$ :

$$
L^{\xi}=p \cdot \hat{H}+X^{\xi}, \quad X^{\xi}=i \sum_{\rho \in \Delta_{+}} g_{|\rho|}(\rho \cdot \hat{H})\left(x(\rho \cdot q)-x\left(\rho^{\vee} \cdot \hat{H} \xi\right)\right) \hat{s}_{\rho},
$$

in which the function $x$ are given in Table I for the degenerate potentials. With the same $M$ operator as before, the quantum equations of motion can be written in a matrix form:

$$
\frac{d}{d t} L^{\xi}=i\left[\mathcal{H}, L^{\xi}\right]=\left[L^{\xi}, M\right]
$$

In other words, the $\xi$ dependent part decouples. This allows us to define a one parameter family of conserved quantities

$$
{ }^{\xi} Q_{n}=\operatorname{Ts}\left(L^{\xi}\right)^{n}, \quad n=1,2, \ldots
$$


which turns out to be a $\xi$ dependent sum of $Q_{n}$ and the lower order conserved quantities $Q_{m}, m<n$. A special limit $\xi \rightarrow-i \infty$ in the trigonometric models provides a convenient combination which allows easy proof of involution in the $A_{r}$ model. (In the rational model, the limit reduces to the Lax pair without spectral parameter. In the hyperbolic models this limit is ill-defined.) In the rest of this subsection we consider only the trigonometric potential models. Let us denote the limiting $L^{\xi}$ operator by $L^{\infty}$ which reads

$$
L^{\infty}=L+a \sum_{\rho \in \Delta_{+}} g_{|\rho|}|\rho \cdot \hat{H}| \hat{s}_{\rho}
$$

and the corresponding $\ell$ operators are given by

$$
\ell_{\mu}^{\infty}=\ell_{\mu}+t_{\mu}, \quad t_{\mu}=a \sum_{\rho \in \Delta_{+}} g_{|\rho|}|\rho \cdot \mu| \check{s}_{\rho}
$$

For the $A_{r}$ model in the vector representation $\mathcal{R}=\left\{\mu_{j} \in \mathbf{R}^{r}, j=1, \ldots, r+1\right\}$ with the standard normalisation of roots $\rho^{2}=2$, the above expression simplifies to

$$
\ell_{j}^{\infty}=\ell_{j}+t_{j}, \quad t_{j}=a g \sum_{k \neq j} \check{s}_{j k},
$$

in which as before we abbreviate $\ell_{\mu_{j}}$ as $\ell_{j}$ and $\check{s}_{j k} \equiv \check{s}_{\mu_{j}-\mu_{k}}$. These are the operators introduced in [15], $\ell_{j} \leftrightarrow \pi_{j}, \ell_{j}^{\infty} \leftrightarrow \tilde{\pi}_{j}$. The Lax operator with the spectral parameter gives an 'explanation' for the rather ad hoc introduction of $\tilde{\pi}_{j}$. It is straightforward to show

$$
\left[\ell_{j}, \ell_{k}\right]=\left[t_{j}, t_{k}\right]
$$

which leads to

$$
\left[\ell_{j}^{\infty}, \ell_{k}^{\infty}\right]=\left[\ell_{j}^{\infty}, t_{k}\right]+\left[t_{j}, \ell_{k}^{\infty}\right]=2 a g\left[\ell_{j}^{\infty}, \check{s}_{j k}\right]=-2 a g\left[\ell_{k}^{\infty}, \check{s}_{j k}\right]
$$

This has the same structure as (8.12) in the previous subsection. By repeating the same argument we arrive at 15

$$
\left[{ }^{\infty} Q_{n},{ }^{\infty} Q_{m}\right]=0, \quad n, m=1, \ldots, r, \quad{ }^{\infty} Q_{n}=\sum_{j=1}^{r}\left(\ell_{j}^{\infty}\right)^{n},
$$

which then imply the involution of the conserved quantities obtained from the original $L$ operator

$$
\left[Q_{n}, Q_{m}\right]=0, \quad n, m=1, \ldots, r
$$




\section{Summary and comments}

We have discussed various issues related to quantum integrability of Calogero-Moser models based on all root systems. These are construction of quantum conserved quantities and a unified proof of their involution, the relationship between the Lax pair and the differentialreflection (Dunkl) operators formalisms, construction of excited states by creation operators, etc. They are mainly generalisations of the results known for the models based on $A_{r}$ root systems, which are shown to apply to the models based on any root systems. There are some interesting works discussing the integrability issues of the models based on other classical root systems and the exceptional ones including the non-crystallographic models [32, [35]- 38].

Here we list some comments on interesting issues which are not treated in this paper. The structure and properties of the eigenfunctions of the trigonometric potential models, which are generalisations of the Jack polynomials [25]-28], will be discussed in future publications. A comprehensive treatment of Liouville integrability of rational models with harmonic force is wanted. Our starting point, the factorised Hamiltonian (2.5) for degenerate potential models, is closely related with supersymmetry and shape invariance [39, 38]. Further investigation in this direction is a future problem. It is a great challenge to formulate various aspects of quantum Calogero-Moser models with elliptic potentials; Lax pair, the differential-reflection operators [40, 41], conserved quantities, supersymmetry and excited states wavefunctions.

\section{Acknowledgements}

We thank K. Takasaki, S. Odake and P. Ghosh for useful discussion. This work is partially supported by the Grant-in-aid from the Ministry of Education, Science and Culture, priority area (\#707) "Supersymmetry and unified theory of elementary particles". S. P. K. and A. J. P. are supported by the Japan Society for the Promotion of Science.

\section{Appendix A: Root Systems}

In this Appendix we recapitulate the rudimentary facts of the root systems and reflections to be used in the main text. The set of roots $\Delta$ is invariant under reflections in the hyperplane perpendicular to each vector in $\Delta$. In other words,

$$
s_{\alpha}(\beta) \in \Delta, \quad \forall \alpha, \beta \in \Delta,
$$


where

$$
s_{\alpha}(\beta)=\beta-\left(\alpha^{\vee} \cdot \beta\right) \alpha, \quad \alpha^{\vee} \equiv 2 \alpha /|\alpha|^{2} .
$$

The set of reflections $\left\{s_{\alpha}, \alpha \in \Delta\right\}$ generates a group $G_{\Delta}$, known as a Coxeter group, or finite reflection group. The orbit of $\beta \in \Delta$ is the set of root vectors resulting from the action of the Coxeter group on it. The set of positive roots $\Delta_{+}$may be defined in terms of a vector $U \in \mathbf{R}^{r}$, with $\alpha \cdot U \neq 0, \forall \alpha \in \Delta$, as those roots $\alpha \in \Delta$ such that $\alpha \cdot U>0$. Given $\Delta_{+}$, there is a unique set of $r$ simple roots $\Pi=\left\{\alpha_{j}, j=1, \ldots, r\right\}$ defined such that they span the root space and the coefficients $\left\{a_{j}\right\}$ in $\beta=\sum_{j=1}^{r} a_{j} \alpha_{j}$ for $\beta \in \Delta_{+}$are all non-negative. The highest root $\alpha_{h}$, for which $\sum_{j=1}^{r} a_{j}$ is maximal, is then also determined uniquely. The subset of reflections $\left\{s_{\alpha}, \alpha \in \Pi\right\}$ in fact generates the Coxeter group $G_{\Delta}$. The products of $s_{\alpha}$, with $\alpha \in \Pi$, are subject solely to the relations

$$
\left(s_{\alpha} s_{\beta}\right)^{m(\alpha, \beta)}=1, \quad \alpha, \beta \in \Pi .
$$

The interpretation is that $s_{\alpha} s_{\beta}$ is a rotation in some plane by $2 \pi / m(\alpha, \beta)$. The set of positive integers $m(\alpha, \beta)$ (with $m(\alpha, \alpha)=1, \forall \alpha \in \Pi$ ) uniquely specify the Coxeter group. The weight lattice $\Lambda(\Delta)$ is defined as the $\mathbf{Z}$-span of the fundamental weights $\left\{\lambda_{j}\right\}, j=1, \ldots, r$, defined by

$$
\alpha_{j}^{\vee} \cdot \lambda_{k}=\delta_{j k}, \quad \alpha_{j} \in \Pi .
$$

The root systems for finite reflection groups may be divided into two types: crystallographic and non-crystallographic. Crystallographic root systems satisfy the additional condition

$$
\alpha^{\vee} \cdot \beta \in \mathbf{Z}, \quad \forall \alpha, \beta \in \Delta,
$$

which implies that the $\mathbf{Z}$-span of $\Pi$ is a lattice in $\mathbf{R}^{r}$ and contains all roots in $\Delta$. We call this the root lattice, which is denoted by $L(\Delta)$. These root systems are associated with simple Lie algebras: $\left\{A_{r}, r \geq 1\right\},\left\{B_{r}, r \geq 2\right\},\left\{C_{r}, r \geq 2\right\},\left\{D_{r}, r \geq 4\right\}, E_{6}, E_{7}, E_{8}, F_{4}$ and $G_{2}$. The Coxeter groups for these root systems are called Weyl groups. The remaining noncrystallographic root systems are $H_{3}, H_{4}$, whose Coxeter groups are the symmetry groups of the icosahedron and four-dimensional 600-cell, respectively, and the dihedral group of order $2 m,\left\{I_{2}(m), m \geq 4\right\}$. 


\section{Appendix B: Conserved quantities}

Here we list for each root system how the full set of independent conserved quantities are obtained by choosing proper representations of the Lax pair. We choose those of the lowest dimensionality for the convenience of practical calculation. Of course there are many other choices of representations giving equally good sets of conserved quantities. The independence of the conserved quantities can be easily verified by considering the free limit: $g_{|\rho|} \rightarrow 0$.

1. $A_{r}$ : For all powers, the vector representation $(r+1$ dimensions $)$ is enough.

2. $B_{r}$ : For all powers, the representation consisting of short $\operatorname{roots}\left\{ \pm e_{j}: j=1, \ldots, r\right\}$, ( $2 r$ dimensions) is enough. Here we adopt the following explicit parametrisation of the $B_{r}$ root system:

$$
B_{r} \text { root system : } \Delta=\left\{ \pm e_{j} \pm e_{k}, \pm e_{j}, \quad j, k=1, \ldots, r \mid e_{j} \in \mathbf{R}^{r}, e_{j} \cdot e_{k}=\delta_{j k}\right\}
$$

3. $C_{r}$ : For all powers, the representation consisting of long roots $\left\{ \pm 2 e_{j}: j=1, \ldots, r\right\}$, ( $2 r$ dimensions) is enough. The following parametrisation of the root system is used:

$$
C_{r} \text { root system : } \Delta=\left\{ \pm e_{j} \pm e_{k}, \pm 2 e_{j}, \quad j, k=1, \ldots, r \mid e_{j} \in \mathbf{R}^{r}, e_{j} \cdot e_{k}=\delta_{j k}\right\}
$$

4. $D_{r}$ : For all even powers, the vector representation ( $2 r$ dimensions) is enough. For the additional one occurring at power $r$, the (anti)-spinor representation $\left(2^{r-1}\right.$ dimensions) would be necessary. They are minimal representations.

5. $E_{6}$ : For all powers, the $\mathbf{2 7}$ (or $\overline{\mathbf{2 7}}$ ) dimensional representation of the Lie algebra is enough. They are minimal representations.

6. $E_{7}$ : For all powers, the $\mathbf{5 6}$ dimensional representation of the Lie algebra is enough. This is a minimal representation.

7. $E_{8}$ : For all powers, the 240 dimensional representation consisting of all the roots is enough. This is not the same as the adjoint representation of the Lie algebra.

8. $F_{4}$ : For all powers, either of the 24 dimensional representation consisting of all the long roots or the short roots is enough. These are not Lie algebra representations.

9. $G_{2}$ : For all powers, either of the 6 dimensional representations consisting of all the long roots or the short roots is enough. These are not Lie algebra representations. 
10. $I_{2}(m)$ : For both powers 2 and $m$, the representation consisting of $V_{m}$ is enough. Here, $V_{m}$ is the set of vectors with 'half" angles of the roots (see (2.10)) given by

$$
V_{m}=\left\{v_{j}=(\cos ((2 j-1) \pi / 2 m), \sin ((2 j-1) \pi / 2 m)) \in \mathbf{R}^{2} \mid j=1, \ldots, m\right\}
$$

11. $H_{3}$ : For all powers, the representation consisting of all the 30 roots is enough.

12. $H_{4}$ : For all powers, the representation consisting of all the 120 roots is enough.

\section{References}

[1] F. Calogero, "Solution of the one-dimensional $N$-body problem with quadratic and/or inversely quadratic pair potentials", J. Math. Phys. 12 (1971) 419-436.

[2] B. Sutherland, "Exact results for a quantum many-body problem in one-dimension. II", Phys. Rev. A5 (1972) 1372-1376.

[3] J. Moser, "Three integrable Hamiltonian systems connected with isospectral deformations", Adv. Math. 16 (1975) 197-220; J. Moser, "Integrable systems of non-linear evolution equations", in Dynamical Systems, Theory and Applications; J. Moser, ed., Lecture Notes in Physics 38 (1975), Springer-Verlag; F. Calogero, C. Marchioro and O. Ragnisco, "Exact solution of the classical and quantal one-dimensional many body problems with the two body potential $V_{a}(x)=g^{2} a^{2} / \sinh ^{2} a x "$, Lett. Nuovo Cim. 13 (1975) 383-387; F. Calogero, "Exactly solvable one-dimensional many body problems", Lett. Nuovo Cim. 13 (1975) 411-416.

[4] M. A. Olshanetsky and A. M. Perelomov, "Completely integrable Hamiltonian systems connected with semisimple Lie algebras", Inventions Math. 37 (1976), 93-108.

[5] M. A. Olshanetsky and A. M. Perelomov, "Quantum integrable systems related to Lie algebras", Phys. Rep. 94 (1983) 313-404.

[6] A. J. Bordner, E. Corrigan and R. Sasaki, "Calogero-Moser models I: a new formulation", Prog. Theor. Phys. 100 (1998) 1107-1129, hep-th/9805106; A. J. Bordner, R. Sasaki and K. Takasaki, "Calogero-Moser models II: symmetries and foldings", Prog. Theor. Phys. 101 (1999) 487-518, hep-th/9809068; A. J. Bordner and R. Sasaki, "Calogero-Moser models III: elliptic potentials and twisting", Prog. Theor. 
Phys. 101 (1999) 799-829, hep-th/9812232; S. P. Khastgir, R. Sasaki and K. Takasaki, "Calogero-Moser Models IV: Limits to Toda theory", Prog. Theor. Phys. 102 (1999), 749-776, hep-th/9907102.

[7] A. J. Bordner, E. Corrigan and R. Sasaki, "Generalised Calogero-Moser models and universal Lax pair operators", Prog. Theor. Phys. 102 (1999) 499-529, hep-th/9905011.

[8] E. D'Hoker and D.H.Phong, "Calogero-Moser Lax pairs with spectral parameter for general Lie algebras", Nucl. Phys. B530 (1998) 537-610, hep-th/9804124.

[9] A. J. Bordner, N. S. Manton and R. Sasaki, "Calogero-Moser Models V: Supersymmetry, and Quantum Lax Pair", Prog. Theor. Phys. 103 (2000) 463-487, hep-th/9910033.

[10] C.F.Dunkl, "Differential-difference operators associated to reflection groups", Trans. Amer. Math. Soc. 311 (1989) 167-183; V. M. Buchstaber, G. Felder and A. P. Veselov, "Elliptic Dunkl operators, root systems and functional equations", Duke Math. J. 76 (1994) 885-911.

[11] M. A. Olshanetsky and A. M. Perelomov, "Quantum systems related to root systems, and radial parts of Laplace operators", Funct. Anal. Appl. 12 (1977) 121-128.

[12] A. M. Perelomov, "Algebraic approach to the solution of a one-dimensional model of interacting particles", Theor. Math. Phys. 6 263-282, (1971).

[13] S. Wojciechowski, "Involutive set of integrals for completely integrable many-body problems with pair interaction", Lett. Nuouv. Cim. 18 (1976) 103-107.

[14] M. Lassalle, "Polynômes de Jacobi, généralisés", "Polynômes de Laguerre généralisés", "Polynômes de Hermite généralisés", C. R. Acad. Sci. Paris, t. Sér. I Math. 312 (1991) 425-428, 725-728, 313 (1991) 579-582.

[15] A. P. Polykronakos, "Exchange operator formalism for integrable systems of particles", Phys. Rev. Lett. 69 (1992) 703-705.

[16] B.S. Shastry and B. Sutherland, "Superlax pairs and infinite symmetries in the $1 / r^{2}$ system", Phys. Rev. Lett. 70 (1993) 4029-4033. 
[17] L. Brink, T. H. Hansson and M. A. Vasiliev, "Explicit solution to the $N$ body Calogero problem", Phys. Lett. B286 (1992) 109-111, hep-th/9206049; L. Brink, T. H. Hansson, S. Konstein and M. A. Vasiliev, "The Calogero model: anyonic representation, fermionic extension and supersymmetry", Nucl. Phys. B401 (1993) 591-612, hep-th/9302023; L. Brink, A. Turbiner and N. Wyllard "Hidden algebras of the (super) Calogero and Sutherland models", J. Math. Phys. 39 (1998) 1285-1315, hep-th/9705219.

[18] H. Ujino M. Wadati and K. Hikami, "The quantum Calogero-Moser model: algebraic structures", J. Phys. Soc. Jpn. 62 (1993) 3035-3043; H. Ujino and M. Wadati, "Rodrigues formula for Hi-Jack symmetric polynomials associated with the quantum Calogero model", J. Phys. Soc. Jpn. 65 (1996) 2423-2439, cond-mat/9609041; H. Ujino, "Orthogonal symmetric polynomials associated with the Calogero model", J. Phys. Soc. Jpn 64 (1995) 2703-2706, cond-mat/9706133; A. Nishino, H. Ujino and M. Wadati, "Symmetric Fock space and orthogonal symmetric polynomials associated with the Calogero model", Chaos Solitons Fractals 11 (2000) 657-674, cond-mat/9803284.

[19] K. Sogo, "A simple derivation of multivariable Hermite and Legendre polynomials", J. Phys. Soc. Jpn 65 (1996) 3097-3099; N. Gurappa and P. K. Panigrahi, "Equivalence of the Calogero-Sutherland model to free harmonic oscillators", Phys. Rev. B59 (1999) R2490-R2493, cond-mat/9710035.

[20] S. N. M. Ruijsenaars, "Sytems of Calogero-Moser type", CRM Series in Math. Phys. 1 251-352, Springer, 1999.

[21] G. J. Heckman, "A remark on the Dunkl differential-difference operators", in W. Barker and P. Sally (eds.) "Harmonic analysis on reductive groups", Birkhäuser, Basel (1991).

[22] G. J. Heckman and E. M. Opdam, "Root systems and hypergeometric functions I", Comp. Math. 64 (1987), 329-352; G. J. Heckman, "Root systems and hypergeometric functions II", Comp. Math. 64 (1987), 353-373; E. M. Opdam, "Root systems and hypergeometric functions III", Comp. Math. 67 (1988), 21-49; "Root systems and hypergeometric functions IV", Comp. Math. 67 (1988), 191-209.

[23] D.Z. Freedman and P.F. Mende, "An exactly solvable $N$-particle system in supersymmetric quantum mechanics", Nucl. Phys. 344 (1990) 317-343. 
[24] W. Rühl and A. Turbiner, "Exact solvability of the Calogero and Sutherland models", Mod. Phys. Lett. A10 (1995) 2213-2222, hep-th/9506105; O. Haschke and W. Rühl, "Is it possible to construct exactly solvable models?", Lecture Notes in Physics 539 118-140, Springer, Berlin 2000, hep-th/9809152.

[25] R. Stanley, "Some combinatorial properties of Jack symmetric function", Adv. Math. 77 (1989) 76-115; I. G. Macdonald, Symmetric functions and Hall polynomials", second edition, Oxford University Press, 1995.

[26] L. Lapointe and L. Vinet, "Rodrigues formulas for the Macdonald polynomials", Adv. Math. 130 (1997) 261-279, q-alg/9607025; "Exact operator solution of the CalogeroSutherland model", Commun. Math. Phys. 178 (1996) 425-452, q-alg/9509003.

[27] T. H. Baker and P. J. Forrester, "The Calogero-Sutherland model and generalized classical polynomials", Commun. Math. Phys. 188 (1997) 175-216, solv-int/9608004.

[28] H. Awata, Y. Matsuo, S. Odake and J. Shiraishi, "Excited states of CalogeroSutherland model and singular vectors of $W_{N}$ algebra", Nucl. Phys. B449 (1995) 347374 , hep-th/9503043.

[29] A. Erdélyi et al. "Higher Transcendental Functions" III, McGraw-Hill, New York, 1955.

[30] P. J. Gambardella, "Exact results in quantum many-body systems of interacting particles in many dimensions with $\overline{S U(1,1)}$ as the dynamical group", J. Math. Phys. 16 1172-1187 (1975).

[31] F. Calogero, "Solution of a three body problem in one dimension", J. Math. Phys. 10 (1969) 2191-2196; "Ground state of a one-dimensional N-body problem", J. Math. Phys. 10 (1969) 2197-2200.

[32] J. Wolfes, "On the three-body linear problem with three-body interaction", J. Math. Phys. 15 (1974) 1420-1424; F. Calogero and C. Marchioro, "Exact solution of a onedimensional three-body scattering problem with two-body and/or three-body inversesquare potential", J. Math. Phys. 15 (1974) 1425-1430.

[33] F. A. Berezin, "Laplace operators on semisimple Lie groups", Tr. Mosk. Mat. Ob-va, 6 (1957) 371-463. 
[34] I. M. Krichever, "Elliptic solutions of the Kadomtsev-Petviashvili equation and integrable systems of particles", Funct. Anal. Appl. 14 (1980) 282-289.

[35] M. Rosenbaum, A. Turbiner and A. Capella, "Solvability of the $g_{2}$ integrable system", Int. J. Mod. Phys. A13 (1998) 3885-3904, solv-int/9707005; N. Gurappa, A. Khare and P. K. Panigrahi, "Connection between Calogero-Marchioro-Wolfes type few-body models and free oscillators", cond-mat/9804207.

[36] O. Haschke and W. Rühl, "An exactly solvable model of the Calogero type for the icosahedral group", Mod. Phys. Lett. A13 (1998) 3109-3122, hep-th/9811011; “The construction of trigonometric invariants for Weyl groups and the derivation of corresponding exactly solvable Sutherland models ", Modern Phys. Lett. A14 (1999) 937949, math-ph/9904002.

[37] S. Kakei, "Common algebraic structure for the Calogero-Sutherland models", J. Phys. A29 (1996) L619-L624; "An orthogonal basis for the $B_{N}$-type Calogero model", J. Phys. A30 (1997) L535-L541.

[38] P. K. Ghosh, A. Khare and M. Sivakumar, "Supersymmetry, shape invariance and solvability of $A_{N-1}$ and $B C_{N}$ Calogero-Sutherland model", Phys. Rev. A58 (1998) 821825, cond-mat/9710206; U. Sukhatme and A. Khare, "Comment on self-isospectral periodic potentials and supersymmetric quantum mechanics", quant-ph/9902072.

[39] C. Efthimiou and D. Spector, "Shape invariance in the Calogero and CalogeroSutherland models", Phys. Rev. A56 (1997) 208-219, quant-ph/9702017.

[40] I. V. Cherednik, "Elliptic quantum many-body problem and double affine KnizhnikZamolodchikov equation", Comm. Math. Phys. 169 (1995) 441-461.

[41] T. Oshima and H. Sekiguchi, "Commuting families of differential operators invariant under the action of a Weyl group", J. Math. Sci. Univ. Tokyo 2 (1995) 1-75. 